ESAIM: COCV 20 (2014) 547-575

DOI: $10.1051 / \mathrm{cocv} / 2013075$
ESAIM: Control, Optimisation and Calculus of Variations

www.esaim-cocv.org

\title{
CONTROLLABILITY OF A SIMPLIFIED MODEL OF FLUID-STRUCTURE INTERACTION *
}

\author{
S. ERVEDOZA ${ }^{1}$ AND M. VANNINATHAN ${ }^{1}$
}

\begin{abstract}
This article aims at studying the controllability of a simplified fluid structure interaction model derived and developed in [C. Conca, J. Planchard and M. Vanninathan, RAM: Res. Appl. Math. John Wiley \& Sons Ltd., Chichester (1995); J.-P. Raymond and M. Vanninathan, ESAIM: COCV 11 (2005) 180-203; M. Tucsnak and M. Vanninathan, Systems Control Lett. 58 (2009) 547-552]. This interaction is modeled by a wave equation surrounding a harmonic oscillator. Our main result states that, in the radially symmetric case, this system can be controlled from the outer boundary. This improves previous results [J.-P. Raymond and M. Vanninathan, ESAIM: COCV 11 (2005) 180-203; M. Tucsnak and M. Vanninathan, Systems Control Lett. 58 (2009) 547-552]. Our proof is based on a spherical harmonic decomposition of the solution and the so-called lateral propagation of the energy for $1 \mathrm{~d}$ waves.
\end{abstract}

Mathematics Subject Classification. 93B05, 93B07, 93C20, 74F10.

Received March 26, 2013. Revised August 13, 2013.

Published online March 28, 2014.

\section{INTRODUCTION}

In this work, we consider a simplified fluid structure interaction model derived and developed in $[6,18,20]$, constituted by a wave equation surrounding an harmonic oscillator.

The model. Let $\omega$ and $\Omega$ be smooth bounded simply connected domains of $\mathbb{R}^{2}$ such that $\bar{\omega} \subset \Omega$, and set $\mathcal{O}=\Omega \backslash \bar{\omega}$. This corresponds to the geometric configuration represented in Figure 1 .

Keywords and phrases. Controllability, observability, fluid-structure interaction.

* The first author is partially supported by the Agence Nationale de la Recherche (ANR, France), Project CISIFS number NT09-437023. The second author is supported by CEFIPRA within the Project 3701-1 Control of Systems of Partial Differential Equations. He also thanks French CNRS and Institut de Mathématiques de Toulouse for hospitality and support for his stay in France during which this work was carried out. Both authors acknowledge the support of the Indo-French Centre for Applied Mathematics (IFCAM).

1 Institut de Mathématiques de Toulouse ; UMR5219; Université de Toulouse ; CNRS; UPS IMT, F-31062 Toulouse Cedex 9,

France. ervedoza@math.univ-toulouse.fr; vanni@math.tifrbng.res.in 


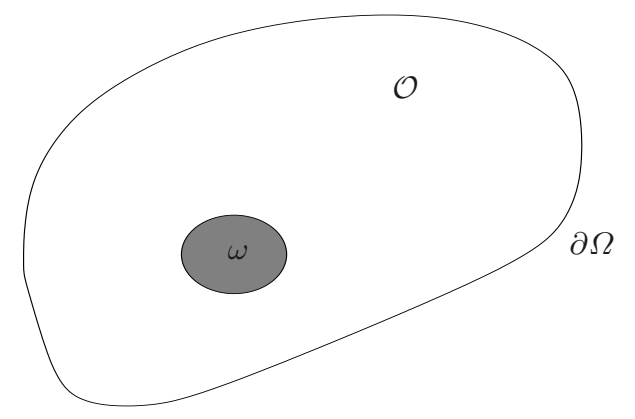

FiguRE 1. The geometric setting.

We are considering the following model:

$$
\begin{cases}y^{\prime \prime}-\Delta y=f, & (t, x) \in(0, T) \times \mathcal{O}, \\ y(t, x)=0, & (t, x) \in(0, T) \times \partial \Omega, \\ \partial_{n} y(t, x)=s^{\prime} \cdot \boldsymbol{n}, & (t, x) \in(0, T) \times \partial \omega, \\ s^{\prime \prime}+s=-\int_{\partial \omega} y^{\prime} \boldsymbol{n} \mathrm{d} \sigma, & t \in(0, T), \\ \left(y(0, \cdot), y^{\prime}(0, \cdot)\right)=\left(y_{0}, y_{1}\right), & \\ \left(s(0), s^{\prime}(0)\right)=\left(s_{0}, s_{1}\right) . & \end{cases}
$$

Here, $\boldsymbol{n}$ denotes the unit outward normal vector on $\mathcal{O}$. The function $y$ represents the velocity potential of the fluid, the function $s\left(\in \mathbb{R}^{2}\right)$ corresponds to the displacement of an oscillator located in $\omega$, and the function $f(t, x)$ is an external force, acting on the fluid and thus localized in $\mathcal{O}$. Here, the prime denotes differentiation with respect to the time variable.

Details on the derivation of (1.1) can be found in [6], p. 96. In particular, model (1.1) corresponds to an inviscid slightly inhomogeneous compressible fluid when the velocity of the fluid is small and assumed to derive from a potential $y$. Accordingly, it may be more relevant to consider Neumann boundary conditions for $y$ on the external boundary $\partial \Omega$. This issue will be discussed in Section 3.6.

The interaction between the wave equation and the oscillator takes place through the boundary conditions on $\partial \omega$ and the source term in the equation in $s$. Actually, we could also handle an equation of the form $s^{\prime \prime}+A_{0} s=-\int_{\partial \omega} y^{\prime} \boldsymbol{n} \mathrm{d} \sigma$ with $A_{0}$ a self-adjoint positive definite $2 \times 2$ matrix for the oscillator, but this would not yield any particular difficulty and we thus choose $A_{0}=I$ to simplify the notations.

Before going further, let us briefly present the Cauchy theory for system (1.1). In order to do that properly, we define the space $H_{\text {ext }}^{1}(\mathcal{O})$ by

$$
H_{\text {ext }}^{1}(\mathcal{O})=\left\{y \in H^{1}(\mathcal{O}), \quad y_{\mid \partial \Omega}=0\right\} .
$$

Then, according to [18], Theorem 2.1, given an initial data $\left(y_{0}, y_{1}, s_{0}, s_{1}\right) \in H_{\text {ext }}^{1}(\mathcal{O}) \times L^{2}(\mathcal{O}) \times\left(\mathbb{R}^{2}\right)^{2}$ and source term $f \in L^{1}\left(0, T ; L^{2}(\mathcal{O})\right)$, system (1.1) has a unique solution $(y, s)$ lying in $C^{0}\left([0, T] ; H_{\text {ext }}^{1}(\mathcal{O})\right) \cap$ $C^{1}\left([0, T] ; L^{2}(\mathcal{O})\right) \times C^{1}\left([0, T] ; \mathbb{R}^{2}\right)$.

Note that this is the natural space since, at least formally, solutions $(y, s)$ of $(1.1)$ have an energy

$$
E(t)=\frac{1}{2} \int_{\mathcal{O}}\left(|\nabla y(t)|^{2}+\left|\partial_{t} y(t)\right|^{2}\right) \mathrm{d} x+\frac{1}{2}\left(|s(t)|^{2}+\left|s^{\prime}(t)\right|^{2}\right),
$$

which satisfies

$$
\frac{\mathrm{d} E}{\mathrm{~d} t}=\int_{\mathcal{O}} \partial_{t} y(t) f(t) \mathrm{d} x
$$

In particular, when $f=0$, the energy of solutions $(y, s)$ of $(1.1)$ is constant in time. 
The control problem. In this article, we focus on the null-controllability property of system (1.1) when the control is an external force $f$ localized in some open subset $\mathcal{C}$ of $\mathcal{O}$. To be more precise, we consider the following controlled system:

$$
\begin{cases}y^{\prime \prime}-\Delta y=\chi_{\mathcal{C}} f, & (t, x) \in(0, T) \times \mathcal{O}, \\ y(t, x)=0, & (t, x) \in(0, T) \times \partial \Omega, \\ \partial_{n} y(t, x)=s^{\prime} \cdot \boldsymbol{n}, & (t, x) \in(0, T) \times \partial \omega, \\ s^{\prime \prime}+s=-\int_{\partial \omega} y^{\prime} \boldsymbol{n} \mathrm{d} \sigma, & t \in(0, T), \\ \left(y(0, \cdot), y^{\prime}(0, \cdot)\right)=\left(y_{0}, y_{1}\right), & \\ \left(s(0), s^{\prime}(0)\right)=\left(s_{0}, s_{1}\right), & \end{cases}
$$

where $f$ is the control function, and $\chi_{\mathcal{C}}$ denotes the indicator function of $\mathcal{C}$.

More precisely, we ask if there exists a time $T>0$ such that for any $\left(y_{0}, y_{1}, s_{0}, s_{1}\right) \in H_{\text {ext }}^{1}(\mathcal{O}) \times L^{2}(\mathcal{O}) \times\left(\mathbb{R}^{2}\right)^{2}$, one can find a control function $f \in L^{2}((0, T) \times \mathcal{C})$ such that the corresponding solution $(y, s)$ of $(1.4)$ satisfies

$$
\left(y(T), y^{\prime}(T), s(T), s^{\prime}(T)\right)=(0,0,0,0) \in H_{\text {ext }}^{1}(\mathcal{O}) \times L^{2}(\mathcal{O}) \times\left(\mathbb{R}^{2}\right)^{2} .
$$

In that case, we say that system (1.4) is null-controllable at time $T$.

Note that, due to the time-reversibility of system (1.4), one easily checks that the null-controllability of (1.4) at time $T$ is equivalent to the exact controllability of (1.4) in time $T$, which states that for any initial and target states $\left(y_{0}, y_{1}, s_{0}, s_{1}\right),\left(y_{0}^{T}, y_{1}^{T}, s_{0}^{T}, s_{1}^{T}\right)$ in $H_{\text {ext }}^{1}(\mathcal{O}) \times L^{2}(\mathcal{O}) \times\left(\mathbb{R}^{2}\right)^{2}$, there exists a control function $f \in L^{2}((0, T) \times \mathcal{C})$ such that the corresponding solution of (1.4) satisfies

$$
\left(y(T), y^{\prime}(T), s(T), s^{\prime}(T)\right)=\left(y_{0}^{T}, y_{1}^{T}, s_{0}^{T}, s_{1}^{T}\right) \in H_{\mathrm{ext}}^{1}(\mathcal{O}) \times L^{2}(\mathcal{O}) \times\left(\mathbb{R}^{2}\right)^{2} .
$$

Hence, in the sequel, we will not distinguish between the null and exact controllability properties.

Of course, the null-controllability property of (1.4) depends on the time $T$ and the set $\mathcal{C}$ in which the control is active. Our goal then is to derive conditions on the time $T$ and the set $\mathcal{C}$ under which we can guarantee null-controllability.

Before stating our results, let us now briefly review previous results on system (1.4) and its controllability property.

In [18], it was proved that when the control function acts as a source term in all the fluid $($ i.e. $\mathcal{C}=\mathcal{O})$, then system (1.4) is exactly controllable in any time $T>0$. Later on in [20], it was proved that when the control is localized in space in the fluid part $\mathcal{O}$ in a neighborhood of both the boundaries $\partial \Omega$ and $\partial \omega$, there exists a time $T$ such that system (1.4) is controllable. Though, this time $T>0$ is not given explicitly in [20]. This is a drawback of the method used in [20], which relies on resolvent estimates [5, 8, 16,21]. This result is not completely satisfactory because one still needs the control region to be localized near the entire outer boundary $\partial \Omega$ and also near the entire interior boundary $\partial \omega$. But such a control may not be possible in real situations.

We also refer to [19], which considers a similar coupling between a structure and a fluid but with a fluid modeled by the Stokes equations. Of course, this yields a completely different model, and the well-posedness and controllability issues are completely different.

Case of a control region in a neighborhood of both boundaries $\partial \Omega$ and $\partial \omega$. Our first main result handles the case in which the control region contains a neighborhood of both boundaries $\partial \Omega$ and $\partial \omega$. Note that this is precisely the setting dealt with in [20] but we are going to provide new estimates on the time of controllability.

We suppose that there exists $\varepsilon>0$ such that

$$
\{x \in \mathcal{O}, d(x, \partial \mathcal{O}) \leq \varepsilon\} \subset \mathcal{C} .
$$

This geometric condition is represented in Figure 2. 


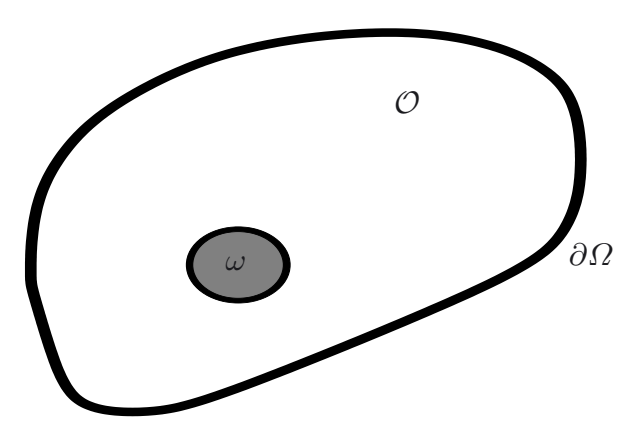

Figure 2. Condition (1.7) means that the control set $\mathcal{C}$ contains the set represented in black, i.e. a neighborhood of both boundaries $\partial \Omega$ and $\partial \omega$.

We also assume that the wave equation in domain $\mathcal{O}$ with homogeneous Dirichlet boundary conditions is null-controllable at time $T_{0}>0$ when the control acts on $\mathcal{C}$.

This means the following: for all $\left(\varphi_{0}, \varphi_{1}\right) \in H_{0}^{1}(\mathcal{O}) \times L^{2}(\mathcal{O})$, there exists a control function $g \in L^{2}\left(\left(0, T_{0}\right) \times \mathcal{C}\right)$ so that the solution $\varphi$ of

$$
\begin{cases}\varphi^{\prime \prime}-\Delta \varphi=\chi_{\mathcal{C}} g, & (t, x) \in\left(0, T_{0}\right) \times \mathcal{O}, \\ \varphi(t, x)=0, & (t, x) \in\left(0, T_{0}\right) \times \partial \mathcal{O} \\ \left(\varphi(0, \cdot), \varphi^{\prime}(0, \cdot)\right)=\left(\varphi_{0}, \varphi_{1}\right) & \end{cases}
$$

satisfies $\left(\varphi\left(T_{0}\right), \varphi^{\prime}\left(T_{0}\right)\right)=(0,0)$.

Note that system (1.8) does not contain any rigid body anymore and simply is a wave equation in $\mathcal{O}$.

According to [1,4], this system is null-controllable in time $T_{0}$ if and only if $\left(\mathcal{C}, \mathcal{O}, T_{0}\right)$ satisfies the Geometric Control Condition of Bardos Lebeau and Rauch. Roughly speaking, this condition states that all the rays of Geometric Optics should meet the control region $\mathcal{C}$ in a time less than $T_{0}$. In our context, these rays of Geometric Optics simply are straight lines bouncing on the boundary $\partial \mathcal{O}$ according to Descartes-Snell's law (Actually, since in the present setting the control set $\mathcal{C}$ contains a neighborhood of both boundaries $\partial \Omega$ and $\partial \omega$, we do not need here to describe the behavior of the rays on the boundary).

Note that, due to Assumption (1.7) and the geometry under consideration, the time $T_{0}$ always exists and is bounded by $\operatorname{diam}(\Omega)$. For instance in the case $\Omega=B\left(0, R_{0}\right)$ and $\omega=B\left(0, r_{0}\right)$, the time $T_{0}$ can be taken to be any time larger than $2 \sqrt{R_{0}^{2}-r_{0}^{2}}$.

Also note that the Hilbert Uniqueness Method provides a control function $g$ in $L^{2}\left(\left(0, T_{0}\right) \times \mathcal{C}\right)$ of minimal $L^{2}\left(\left(0, T_{0}\right) \times \mathcal{C}\right)$-norm which moreover satisfies

$$
\|g\|_{L^{2}\left(\left(0, T_{0}\right) \times \mathcal{C}\right)} \leq C\left\|\left(\varphi_{0}, \varphi_{1}\right)\right\|_{H_{0}^{1}(\mathcal{O}) \times L^{2}(\mathcal{O})} .
$$

We then prove the following:

Theorem 1.1. Assume that $\mathcal{C}$ satisfies Assumption (1.7) and that system (1.8) is null-controllable at time $T_{0}>0$. Then system (1.4) is exactly controllable at time $T_{0}$.

Our argument consists mainly of two things:

- the control result obtained in [18] when the control acts everywhere in the fluid part $\mathcal{O}$;

- the null-controllability of the wave equation (1.8).

The proof of Theorem 1.1 is then reduced to a suitable gluing argument between the two controlled trajectories. Details of the Proof of Theorem 1.1 are given in Section 2. 


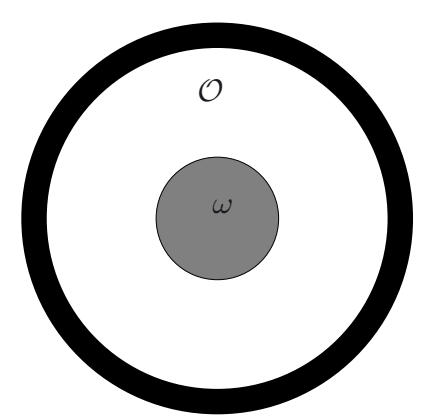

Figure 3. The geometric setting in the radial configuration given by (1.10). Here, the control set $\mathcal{C}$ is represented in black: it is a ring of the form $\mathcal{C}=B\left(0, R_{0}\right) \backslash B\left(0, R_{1}\right)$ localized close to the external boundary.

The radial case. We then focus on the radial case: we assume that there exists $r_{0}, R_{0}, R_{1}$ positive real numbers with $0<r_{0}<R_{1}<R_{0}$ and

$$
\omega=B\left(0, r_{0}\right), \quad \Omega=B\left(0, R_{0}\right), \quad \mathcal{C}=B\left(0, R_{0}\right) \backslash \overline{B\left(0, R_{1}\right)},
$$

where $B(0, r)$ denotes the ball centered at the origin of radius $r>0$. This corresponds to the geometric setting given in Figure 3.

We then prove the following:

Theorem 1.2. Under Assumption (1.10), system (1.4) is exactly controllable in any time

$$
T>2 \sqrt{R_{1}^{2}-r_{0}^{2}}
$$

To our knowledge, Theorem 1.2 is the first control result for system (1.4) which does not require the control to be supported in a neighborhood of $\partial \omega$. The main issue here is to ensure that no energy is trapped near the boundary $\partial \omega$ as the coupled system vibrates according to the model under consideration.

Note however that Theorem 1.2 is restricted to the radial case. As we shall explain in Section 3.4, this assumption can be slightly weakened and the form of $\Omega$ and $\mathcal{C}$ can be relaxed. However, the radial assumption on $\omega$ is completely necessary to our arguments.

Let us also emphasize that condition (1.11) is necessarily sharp due to the speed of propagation of waves. Indeed, following the construction of Ralston in [17], if $T<2 \sqrt{R_{1}^{2}-r_{0}^{2}}$, one can construct solutions of the system (1.1) with source term 0, known as Gaussian beams, which are localized outside $(0, T) \times \mathcal{C}$. By duality, this provides an impediment for the controllability of (1.4), see Section 4.

As explained in Section 3.1, following the Hilbert Uniqueness Method of Lions [13], the control result stated in Theorem 1.2 is based on the observability of the adjoint equation

$$
\begin{cases}\varphi^{\prime \prime}-\Delta \varphi=0, & (t, x) \in(0, T) \times \mathcal{O}, \\ \varphi(t, x)=0, & (t, x) \in(0, T) \times \partial \Omega, \\ \partial_{n} \varphi(t, x)=w^{\prime} \cdot \boldsymbol{n}, & (t, x) \in(0, T) \times \partial \omega, \\ w^{\prime \prime}+w=-\int_{\partial \omega} \varphi^{\prime} \boldsymbol{n} \mathrm{d} \sigma, & t \in(0, T), \\ \left(\varphi(0, \cdot), \varphi^{\prime}(0, \cdot)\right)=\left(\varphi_{0}, \varphi_{1}\right), & \\ \left(w(0), w^{\prime}(0)\right)=\left(w_{0}, w_{1}\right) . & \end{cases}
$$


Namely, we are going to study the following observability inequality: there exists a constant $C>0$ such that for all $\left(\varphi_{0}, \varphi_{1}, w_{0}, w_{1}\right) \in H_{\text {ext }}^{1}(\mathcal{O}) \times L^{2}(\mathcal{O}) \times\left(\mathbb{R}^{2}\right)^{2}$, the solution $(\varphi, w)$ of $(1.12)$ satisfies

$$
\left\|\left(\varphi_{0}, \varphi_{1}, w_{0}, w_{1}\right)\right\|_{H_{\mathrm{ext}}^{1}(\mathcal{O}) \times L^{2}(\mathcal{O}) \times\left(\mathbb{R}^{2}\right)^{2}}^{2} \leq C_{\mathrm{obs}}^{2} \int_{0}^{T} \int_{\mathcal{C}} \chi_{\mathcal{C}}^{2}\left|\varphi^{\prime}(t, x)\right|^{2} \mathrm{~d} t \mathrm{~d} x .
$$

In order to prove that observability inequality, we strongly use the radial symmetry of the problem, which allows us to introduce polar coordinates and to do a spherical harmonic decomposition. In particular, we show somewhat surprisingly that the interaction between the waves and the oscillator appears only on the first mode of the Laplace-Beltrami operator on the disk, the other ones being completely decoupled from the displacement $s$ of the solid. That way, we are able to derive a very precise description of the fluid structure interaction.

The main issue is then to study a $1 \mathrm{~d}$ wave equation coupled with an oscillator and its observability property. This study is done using the lateral propagation of the energy of 1d waves, which, roughly speaking, consists in exchanging the role played by the time and the space. This technique is rather classical and has been developed extensively in the context of $1 \mathrm{~d}$ semilinear wave equation (see e.g. [22]).

Note also that our method gives a very precise description of the interaction between the fluid and the solid parts, which partly explains the difficulty of removing the radially symmetric assumption on the solid $\omega$. As we shall explain in Section 3.4, using microlocal defect measures as in $[3,4]$, we can remove the spherical assumption on $\Omega$ and $\mathcal{C}$. However, our method fails to apply when the solid part $\omega$ is not a disk.

Outline. Section 2 aims at proving Theorem 1.1. Radially symmetric domains are considered in Section 3. In Section 3.1, we reduce the null controllability problem for (1.4) to the observability inequality (1.13) for the adjoint system (1.12). Working with polar coordinates and spherical harmonics decomposition of the system, we show in Section 3.2 that only the first harmonic mode couples with the solid, while other modes are free of couplings. The problem is thus reduced to proving observability estimates for the individual components of the decomposition, a task we perform in Section 3.3. In this process, we use the principle of lateral propagation of the energy for $1 \mathrm{~d}$ waves to treat the first harmonic mode. Section 3.4 then provides the corresponding control results and some generalizations of it when the control set does not contain a neighborhood of the entire outer boundary. Section 3.5 is devoted to the boundary controllability of the model. Section 3.6 discusses control results when the potential $y$ is assumed to satisfy the Neumann boundary conditions on the external boundary $\partial \Omega$. Finally, in Section 4, we provide the reader with comments and open problems.

\section{Proof of Theorem 1.1}

The goal of this section is to prove Theorem 1.1. In particular, in this section, we will always assume Condition (1.7).

The Proof of Theorem 1.1 is based on the following result, obtained in [18], Theorem 1.1:

Theorem 2.1 ([18]). For any $T>0$, for any $\left(z_{0}, z_{1}, \sigma_{0}, \sigma_{1}\right) \in H_{\text {ext }}^{1}(\mathcal{O}) \times L^{2}(\mathcal{O}) \times\left(\mathbb{R}^{2}\right)^{2}$, there exists a control function $u \in L^{2}((0, T) \times \mathcal{O})$ such that the solution of

$$
\begin{cases}z^{\prime \prime}-\Delta z=u, & (t, x) \in(0, T) \times \mathcal{O}, \\ z(t, x)=0, & (t, x) \in(0, T) \times \partial \Omega, \\ \partial_{n} z(t, x)=\sigma^{\prime} \cdot \boldsymbol{n}, & (t, x) \in(0, T) \times \partial \omega, \\ \sigma^{\prime \prime}+\sigma=-\int_{\partial \omega} z^{\prime} \boldsymbol{n} \mathrm{d} \sigma, & t \in(0, T), \\ \left(z(0, \cdot), z^{\prime}(0, \cdot)\right)=\left(z_{0}, z_{1}\right), & \\ \left(\sigma(0), \sigma^{\prime}(0)\right)=\left(\sigma_{0}, \sigma_{1}\right) & \end{cases}
$$

satisfies the null-controllability requirement

$$
\left(z(T), z^{\prime}(T), \sigma(T), \sigma^{\prime}(T)\right)=(0,0,0,0) .
$$


Besides, there exists a constant $C_{T}$ such that

$$
\|u\|_{L^{2}((0, T) \times \mathcal{O})}+\|z\|_{L^{\infty}\left(0, T ; H_{\mathrm{ext}}^{1}(\mathcal{O})\right)} \leq C_{T}\left\|\left(z_{0}, z_{1}, \sigma_{0}, \sigma_{1}\right)\right\|_{H_{\mathrm{ext}}^{1}(\mathcal{O}) \times L^{2}(\mathcal{O}) \times\left(\mathbb{R}^{2}\right)^{2}} .
$$

We are now in position to prove Theorem 1.1.

Proof of Theorem 1.1. Let $\mathcal{C}$ satisfying (1.7) for some $\varepsilon>0$, and assume that $T_{0}$ is so that $\left(\mathcal{C}, \mathcal{O}, T_{0}\right)$ satisfies the Geometric Control Condition.

Fix $\left(y_{0}, y_{1}, s_{0}, s_{1}\right) \in H_{\text {ext }}^{1}(\mathcal{O}) \times L^{2}(\mathcal{O}) \times\left(\mathbb{R}^{2}\right)^{2}$. Then, according to Theorem 2.1, setting $\left(z_{0}, z_{1}, \sigma_{0}, \sigma_{1}\right)=$ $\left(y_{0}, y_{1}, s_{0}, s_{1}\right)$, there exists a control function $u \in L^{2}\left(\left(0, T_{0}\right) \times \mathcal{O}\right)$ so that the solution $(z, \sigma)$ of $(2.1)$ satisfies $(2.2)$.

Besides, one has

$$
\|u\|_{L^{2}\left(\left(0, T_{0}\right) \times \mathcal{O}\right)}+\|z\|_{L^{\infty}\left(0, T_{0} ; H_{\mathrm{ext}}^{1}(\mathcal{O})\right)} \leq C\left\|\left(y_{0}, y_{1}, s_{0}, s_{1}\right)\right\|_{H_{\mathrm{ext}}^{1}(\mathcal{O}) \times L^{2}(\mathcal{O}) \times\left(\mathbb{R}^{2}\right)^{2}} .
$$

Now, let $\eta_{1}=\eta_{1}(x)$ be a smooth function in $\mathcal{O}$ taking value one on $\{x \in \mathcal{O}, d(x, \partial \omega) \leq \varepsilon / 2\}$ and vanishing identically on $\{x \in \mathcal{O}, d(x, \partial \omega) \geq \varepsilon\}$.

Then $\left(\eta_{1} z, \sigma\right)$ satisfies the system

$$
\begin{cases}\left(\eta_{1} z\right)^{\prime \prime}-\Delta\left(\eta_{1} z\right)=\eta_{1} u-2 \nabla \eta_{1} \nabla z-\Delta \eta_{1} z, & (t, x) \in\left(0, T_{0}\right) \times \mathcal{O}, \\ \eta_{1} z(t, x)=0, & (t, x) \in\left(0, T_{0}\right) \times \partial \Omega \\ \partial_{n}\left(\eta_{1} z\right)(t, x)=\sigma^{\prime} \cdot \boldsymbol{n}, & (t, x) \in\left(0, T_{0}\right) \times \partial \omega \\ \sigma^{\prime \prime}+\sigma=-\int_{\partial \omega}\left(\eta_{1} z\right)^{\prime} \boldsymbol{n} \mathrm{d} \sigma, & t \in\left(0, T_{0}\right), \\ \left(\eta_{1} z(0, \cdot),\left(\eta_{1} z\right)^{\prime}(0, \cdot)\right)=\left(\eta_{1} y_{0}, \eta_{1} y_{1}\right), & \\ \left(\sigma(0), \sigma^{\prime}(0)\right)=\left(\sigma_{0}, \sigma_{1}\right), & \\ \left(\eta_{1} z\left(T_{0}, \cdot\right),\left(\eta_{1} z\right)^{\prime}\left(T_{0}, \cdot\right)\right)=(0,0), & \\ \left(\sigma\left(T_{0}\right), \sigma^{\prime}\left(T_{0}\right)\right)=(0,0), & \end{cases}
$$

We then define $\eta_{2}=\eta_{2}(x)$ as a smooth function in $\mathcal{O}$ taking value one on $\{x \in \mathcal{O}, d(x, \partial \omega) \leq \varepsilon / 4\}$ and vanishing identically on $\{x \in \mathcal{O}, d(x, \partial \omega) \geq \varepsilon / 2\}$.

Since the wave equation (1.8) is assumed to be null-controllable in time $T_{0}$, setting $\left(\varphi_{0}, \varphi_{1}\right)=\left(\left(1-\eta_{2}\right) y_{0},(1-\right.$ $\left.\left.\eta_{2}\right) y_{1}\right) \in H_{0}^{1}(\mathcal{O}) \times L^{2}(\mathcal{O})$ there exists a control function $g \in L^{2}\left(\left(0, T_{0}\right) \times \mathcal{C}\right)$ that steers the solution $\varphi$ of $(1.8)$ from $\left(\varphi_{0}, \varphi_{1}\right)$ to $(0,0)$ in a time $T_{0}$ satisfying (1.9) and, following,

$$
\begin{aligned}
\|g\|_{L^{2}\left(\left(0, T_{0}\right) \times \mathcal{C}\right)}+\|\varphi\|_{L^{\infty}\left(0, T_{0} ; H_{0}^{1}(\mathcal{O})\right)} & \leq C\left\|\left(\varphi_{0}, \varphi_{1}\right)\right\|_{H_{0}^{1}(\mathcal{O}) \times L^{2}(\mathcal{O})} \\
& \leq C\left\|\left(y_{0}, y_{1}\right)\right\|_{H_{\text {ext }}^{1}(\mathcal{O}) \times L^{2}(\mathcal{O})} .
\end{aligned}
$$

Moreover, $\left(1-\eta_{1}\right) \varphi$ satisfies

$$
\begin{cases}\left(\left(1-\eta_{1}\right) \varphi\right)^{\prime \prime}-\Delta\left(\left(1-\eta_{1}\right) \varphi\right)= & \left(1-\eta_{1}\right) \chi_{\mathcal{c}} g+2 \nabla \eta_{1} \nabla \varphi+\Delta \eta_{1} \varphi \\ \left(\left(1-\eta_{1}\right) \varphi\right)(t, x)=0, & (t, x) \in\left(0, T_{0}\right) \times \mathcal{O} \\ \partial_{n}\left(\left(1-\eta_{1}\right) \varphi\right)(t, x)=0, & (t, x) \in\left(0, T_{0}\right) \times \partial \mathcal{O} \\ & \left(0, T_{0}\right) \times \partial \omega\end{cases}
$$

with initial and final data

$$
\left\{\begin{array}{l}
\left(\left(1-\eta_{1}\right) \varphi(0, \cdot),\left(1-\eta_{1}\right) \varphi^{\prime}(0, \cdot)\right)=\left(\left(1-\eta_{1}\right) y_{0},\left(1-\eta_{1}\right) y_{1}\right) \\
\left(\left(1-\eta_{1}\right) \varphi\left(T_{0}, \cdot\right),\left(1-\eta_{1}\right) \varphi^{\prime}\left(T_{0}, \cdot\right)\right)=(0,0)
\end{array}\right.
$$

where we have used that, due to the conditions on the support of $\eta_{1}$ and $\eta_{2},\left(1-\eta_{1}\right)\left(1-\eta_{2}\right)=\left(1-\eta_{1}\right)$. 
Note that $\left(1-\eta_{1}\right) \varphi$ satisfies null Dirichlet and Neumann boundary conditions on the interface $\partial \omega$. In particular, setting $y=\eta_{1} z+\left(1-\eta_{1}\right) \varphi$ and $s=\sigma$, one immediately gets that $(y, s)$ satisfies (1.4) with initial data $\left(y_{0}, y_{1}, s_{0}, s_{1}\right)$, and control function

$$
\chi_{\mathcal{C}} f=\eta_{1} u-2 \nabla \eta_{1} \nabla z-\Delta \eta_{1} z+\left(1-\eta_{1}\right) \chi_{\mathcal{C}} g+2 \nabla \eta_{1} \nabla \varphi+\Delta \eta_{1} \varphi .
$$

Indeed, the right hand side terms in (2.9) are all supported in $\mathcal{C}$ due to the conditions on the support of $\eta_{1}$ and (1.7), and each term belongs to $L^{2}\left(\left(0, T_{0}\right) \times \mathcal{C}\right)$ thanks to $(2.4)$ and $(2.6)$.

This concludes the Proof of Theorem 1.1 since by construction, $(y, s)$ solves the null controllability requirement (1.5) with $T=T_{0}$.

\section{The RADially SYMmetric CASE}

In this section, we consider the radially symmetric case and assume the geometry given in (1.10):

$$
\omega=B\left(0, r_{0}\right), \Omega=B\left(0, R_{0}\right), \mathcal{C}=B\left(0, R_{0}\right) \backslash \overline{B\left(0, R_{1}\right)}, \text { with } 0<r_{0}<R_{1}<R_{0} .
$$

To simplify notations, we set $\mathcal{A}\left(r_{a}, r_{b}\right)$ the annulus $B\left(0, r_{b}\right) \backslash \overline{B\left(0, r_{a}\right)}$. We also define $S(0, r)$ as the circle of radius $r>0$.

Note that our assumptions also concern the control region $\mathcal{C}$, which is assumed to be an annulus $\mathcal{C}=$ $\mathcal{A}\left(R_{1}, R_{0}\right)$. In particular, the control region is a neighborhood of the whole external boundary, but not of the internal boundary $S\left(0, r_{0}\right)$.

Now, system (1.4) is rewritten as:

$$
\begin{cases}y^{\prime \prime}-\Delta y=\chi_{R_{1}<|x|<R_{0}} f, & (t, x) \in(0, T) \times \mathcal{A}\left(r_{0}, R_{0}\right), \\ y(t, x)=0, & (t, x) \in(0, T) \times S\left(0, R_{0}\right), \\ \partial_{n} y(t, x)=s^{\prime} \cdot \boldsymbol{n}, & (t, x) \in(0, T) \times S\left(0, r_{0}\right), \\ s^{\prime \prime}+s=-\int_{S\left(0, r_{0}\right)} y^{\prime} \boldsymbol{n} \mathrm{d} \sigma, & t \in(0, T), \\ \left(y(0, \cdot), y^{\prime}(0, \cdot)\right)=\left(y_{0}, y_{1}\right), & \\ \left(s(0), s^{\prime}(0)\right)=\left(s_{0}, s_{1}\right) . & \end{cases}
$$

\subsection{Link between observability and controllability}

In this section, the fact that we are considering the radially symmetric case is irrelevant. We therefore consider the control problem (1.4)-(1.5) and the corresponding adjoint system (1.12).

Let us begin by explaining why equation (1.12) can be seen as the adjoint of (1.4). To this end, let us remark that system (1.4) can be rewritten as a first-order system

$$
\left(\begin{array}{c}
y \\
y^{\prime} \\
s \\
s^{\prime}
\end{array}\right)^{\prime}=A\left(\begin{array}{c}
y \\
y^{\prime} \\
s \\
s^{\prime}
\end{array}\right)+B f
$$

where

$$
A\left(\begin{array}{l}
y_{0} \\
y_{1} \\
s_{0} \\
s_{1}
\end{array}\right)=\left(\begin{array}{c}
y_{1} \\
\Delta y_{0} \\
s_{1} \\
-\int_{\partial \omega} y_{1} \boldsymbol{n} d \sigma-s_{0}
\end{array}\right), \quad B=\left(\begin{array}{c}
0 \\
\chi_{\mathcal{C}} \\
0 \\
0
\end{array}\right) .
$$

The operator $A$ is an unbounded operator defined on the space

$$
X=\left\{\left(\begin{array}{l}
y_{0} \\
y_{1} \\
s_{0} \\
s_{1}
\end{array}\right), \text { with } \begin{array}{l}
y_{0} \in H_{\mathrm{ext}}^{1}(\mathcal{O}), \\
y_{1} \in L^{2}(\mathcal{O}) \\
s_{0} \in \mathbb{R}^{2} \\
s_{1} \in \mathbb{R}^{2}
\end{array}\right\}
$$


and its domain is

$$
\mathcal{D}(A)=\left\{\left(\begin{array}{l}
y_{0} \\
y_{1} \\
s_{0} \\
s_{1}
\end{array}\right), \text { with } \begin{array}{l}
y_{0} \in H^{2}(\mathcal{O}) \cap H_{\text {ext }}^{1}(\mathcal{O}) \\
\partial_{1} y_{0}=s_{1} \cdot \boldsymbol{n} \text { on } \partial \omega \\
s_{0} \in \mathbb{R}^{2}, \quad s_{1} \in \mathbb{R}^{2}
\end{array}\right\}
$$

Of course, $X$ is a Hilbert space when endowed with its natural scalar product

$$
\left\langle\left(\begin{array}{c}
y_{0} \\
y_{1} \\
s_{0} \\
s_{1}
\end{array}\right),\left(\begin{array}{c}
z_{0} \\
z_{1} \\
\sigma_{0} \\
\sigma_{1}
\end{array}\right)\right\rangle_{X}=\int_{\mathcal{O}} \nabla y_{0} \cdot \nabla z_{0}+\int_{\mathcal{O}} y_{1} z_{1}+s_{0} \cdot \sigma_{0}+s_{1} \cdot \sigma_{1}
$$

Besides, one easily checks that $A$ is skew-adjoint with respect to this scalar product. Therefore, system (1.12) is the adjoint of system (1.4) when identifying the dual of $X$ with itself.

Let us also note that the adjoint system (1.12) is conservative: The energy of solutions $(\varphi, w)$ of $(1.12)$, defined by

$$
\begin{aligned}
E(t) & =\frac{1}{2}\left\|\left(\varphi(t), \varphi^{\prime}(t), w(t), w^{\prime}(t)\right)\right\|_{H_{\mathrm{ext}}^{1}(\mathcal{O}) \times L^{2}(\mathcal{O}) \times\left(\mathbb{R}^{2}\right)^{2}}^{2} \\
& =\frac{1}{2} \int_{\mathcal{O}}\left(|\nabla \varphi(t)|^{2}+\left|\varphi^{\prime}(t)\right|^{2}\right)+\frac{1}{2}\left(|w(t)|^{2}+\left|w^{\prime}(t)\right|^{2}\right)
\end{aligned}
$$

is constant in time.

Remark 3.1. Let us emphasize that here we made the choice of identifying $X$ with its dual. This will make our arguments easier since we are going to work only with finite-energy solutions. Other choices are possible, and in particular the one in [18], which consists in identifying the dual of $L^{2}(\mathcal{O})$ with itself. The adjoint system should then be stated in the dual of $H_{\text {ext }}^{1}(\mathcal{O}) \times L^{2}(\mathcal{O}) \times\left(\mathbb{R}^{2}\right)^{2}$, which is not completely obvious to characterize. This requires a careful analysis done in [18] which we shortcut here.

The by-now classical Hilbert Uniqueness Method (HUM) of Lions [13,14] then yields the following result:

Theorem 3.2. Assume that $T>0$ is such that the observability property (1.13) holds for solutions of (1.12). Then system (1.4) is exactly controllable in time $T$ : given any initial data $\left(y_{0}, y_{1}, s_{0}, s_{1}\right) \in H_{\text {ext }}^{1}(\mathcal{O}) \times L^{2}(\mathcal{O}) \times$ $\left(\mathbb{R}^{2}\right)^{2}$, there exists a control function $f \in L^{2}((0, T) \times \mathcal{C})$ such that the solution of $(1.4)$ solves $(1.5)$.

Besides, it can be computed as follows. Let us define the functional $J$ on $H_{\text {ext }}^{1}(\mathcal{O}) \times L^{2}(\mathcal{O}) \times\left(\mathbb{R}^{2}\right)^{2}$ by

$$
J\left(\varphi_{0}, \varphi_{1}, w_{0}, w_{1}\right)=\frac{1}{2} \int_{0}^{T} \int_{\mathcal{C}} \chi_{\mathcal{C}}^{2}\left|\varphi^{\prime}(t, x)\right|^{2} \mathrm{~d} t \mathrm{~d} x+\int_{\mathcal{O}} \nabla \varphi_{0} \cdot \nabla y_{0}+\int_{\mathcal{O}} \varphi_{1} y_{1}+w_{0} \cdot s_{0}+w_{1} \cdot s_{1},
$$

where $(\varphi, s)$ is the solution of $(1.12)$ with initial data $\left(y_{0}, y_{1}, s_{0}, s_{1}\right) \in H_{\mathrm{ext}}^{1}(\mathcal{O}) \times L^{2}(\mathcal{O}) \times\left(\mathbb{R}^{2}\right)^{2}$.

The functional $J$ has a unique minimizer $\left(\tilde{\varphi}_{0}, \tilde{\varphi}_{1}, \tilde{w}_{0}, \tilde{w}_{1}\right)$ in $H_{\text {ext }}^{1}(\mathcal{O}) \times L^{2}(\mathcal{O}) \times\left(\mathbb{R}^{2}\right)^{2}$. Denoting by $(\tilde{\varphi}, \tilde{w})$ the corresponding solution of (1.12), the function

$$
f=\chi_{\mathcal{C}} \tilde{\varphi}^{\prime}, \quad(t, x) \in(0, T) \times \mathcal{C}
$$

is an admissible control function for (1.4) and (1.5).

Moreover, it has minimal $L^{2}((0, T) \times \mathcal{C})$-norm among all admissible controls for (1.4)-(1.5).

Proof. Multiplying the equation of $y$ in (1.4) by $\varphi^{\prime}, \varphi$ being a smooth solution of (1.12) and integrating by parts, we obtain

$$
\int_{0}^{T} \int_{\mathcal{C}} \chi_{\mathcal{C}} f \varphi^{\prime}=-\int_{0}^{T} \int_{\mathcal{O}} y^{\prime} \varphi^{\prime \prime}-\int_{0}^{T} \int_{\mathcal{O}} \nabla y^{\prime} \cdot \nabla \varphi+\left.\int_{\mathcal{O}} \nabla \varphi \cdot \nabla y\right|_{0} ^{T}+\left.\int_{\mathcal{O}} \varphi^{\prime} y^{\prime}\right|_{0} ^{T}-\int_{0}^{T} \int_{\partial \omega} \partial_{n} y \varphi^{\prime} .
$$


But

$$
-\int_{0}^{T} \int_{\mathcal{O}} \nabla y^{\prime} \cdot \nabla \varphi=\int_{0}^{T} \int_{\mathcal{O}} y^{\prime} \Delta \varphi-\int_{0}^{T} \int_{\partial \omega} y^{\prime} \partial_{n} \varphi
$$

Besides, the equations on the boundary $\partial \omega$ in (1.12) and in (1.4) yield

$$
\begin{aligned}
-\int_{\partial \omega} \partial_{n} y \varphi^{\prime}-\int_{\partial \omega} y^{\prime} \partial_{n} \varphi=-\int_{\partial \omega} s^{\prime} \cdot \boldsymbol{n} \varphi^{\prime}-\int_{\partial \omega} y^{\prime} w^{\prime} \cdot \boldsymbol{n} & =-s^{\prime} \cdot\left(\int_{\partial \omega} \varphi^{\prime} \boldsymbol{n}\right)-w^{\prime} \cdot\left(\int_{\partial \omega} y^{\prime} \boldsymbol{n}\right) \\
& =s^{\prime} \cdot\left(w^{\prime \prime}+w\right)+w^{\prime} \cdot\left(s^{\prime \prime}+s\right)=\frac{\mathrm{d}}{\mathrm{d} t}\left(s^{\prime} \cdot w^{\prime}+s \cdot w\right) .
\end{aligned}
$$

Combining these last identities, we get

$$
\int_{0}^{T} \int_{\mathcal{C}} \chi_{\mathcal{C}} f \varphi^{\prime}=\left.\int_{\mathcal{O}} \nabla \varphi \cdot \nabla y\right|_{0} ^{T}+\left.\int_{\mathcal{O}} \varphi^{\prime} y^{\prime}\right|_{0} ^{T}+\left.\left(s^{\prime} \cdot w^{\prime}+s \cdot w\right)\right|_{0} ^{T} .
$$

Therefore, $f$ is an admissible control function for (1.4) if and only if for all $\left(\varphi_{0}, \varphi_{1}, w_{0}, w_{1}\right) \in H_{\text {ext }}^{1}(\mathcal{O}) \times L^{2}(\mathcal{O}) \times$ $\left(\mathbb{R}^{2}\right)^{2}$

$$
0=\int_{0}^{T} \int_{\mathcal{C}} \chi_{\mathcal{C}} f \varphi^{\prime}+\int_{\mathcal{O}} \nabla \varphi_{0} \cdot \nabla y_{0}+\int_{\mathcal{O}} \varphi_{1} y_{1}+s_{1} \cdot w_{1}+s_{0} \cdot w_{0}
$$

If $\left(\tilde{\varphi}_{0}, \tilde{\varphi}_{1}, \tilde{w}_{0}, \tilde{w}_{1}\right) \in H_{\text {ext }}^{1}(\mathcal{O}) \times L^{2}(\mathcal{O}) \times\left(\mathbb{R}^{2}\right)^{2}$ is a minimizer of the functional $J$ defined in (3.3), then the corresponding Euler-Lagrange equation coincides with (3.6) for $f=\chi_{\mathcal{C}} \tilde{\varphi}^{\prime}$, hence $f=\chi_{\mathcal{C}} \tilde{\varphi}^{\prime}$ is an admissible control function for (1.4).

One should then explain that such a minimizer exists. This is precisely due to the observability inequality (1.13) which easily implies that the functional $J$ is strictly convex and coercive. Therefore it has a unique minimizer (since $J$ is convex, this also is the only critical point) $\left(\tilde{\varphi}_{0}, \tilde{\varphi}_{1}, \tilde{w}_{0}, \tilde{w}_{1}\right)$.

We then indicate how to prove that $f=\chi_{\mathcal{C}} \tilde{\varphi}^{\prime}$ is the control function of minimal $L^{2}((0, T) \times \mathcal{C})$-norm. For any control function $f_{1}$, and in particular also for $f$ as in $(3.4)$, one can choose $(\varphi, w)=(\tilde{\varphi}, \tilde{w})$ in (3.6). Subtracting the identities with $f_{1}$ and with $f=\chi_{\mathcal{C}} \tilde{\varphi}^{\prime}$, one obtains

$$
\int_{0}^{T} \int_{\mathcal{C}} \chi_{\mathcal{C}} f \tilde{\varphi}^{\prime}=\int_{0}^{T} \int_{\mathcal{C}} \chi_{\mathcal{C}} f_{1} \tilde{\varphi}^{\prime}
$$

hence

$$
\|f\|_{L^{2}((0, T) \times \mathcal{C})}^{2}=\int_{0}^{T} \int_{\mathcal{C}} f_{1} f \leq\left\|f_{1}\right\|_{L^{2}((0, T) \times \mathcal{C})}\|f\|_{L^{2}((0, T) \times \mathcal{C})} .
$$

This completes the Proof of Theorem 3.2.

In the radially symmetric context we are considering, the adjoint system (1.12) becomes:

$$
\begin{cases}\varphi^{\prime \prime}-\Delta \varphi=0, & (t, x) \in(0, T) \times \mathcal{A}\left(r_{0}, R_{0}\right), \\ \varphi(t, x)=0, & (t, x) \in(0, T) \times S\left(0, R_{0}\right), \\ \partial_{n} \varphi(t, x)=w^{\prime} \cdot \boldsymbol{n}, & (t, x) \in(0, T) \times S\left(0, r_{0}\right), \\ w^{\prime \prime}+w=-\int_{S\left(0, r_{0}\right)} \varphi^{\prime} \boldsymbol{n} \mathrm{d} \sigma, & t \in(0, T), \\ \left(\varphi(0, \cdot), \varphi^{\prime}(0, \cdot)\right)=\left(\varphi_{0}, \varphi_{1}\right), & \\ \left(w(0), w^{\prime}(0)\right)=\left(w_{0}, w_{1}\right), & \end{cases}
$$

and the corresponding observability inequality is the following one: Find $T>0$ for which there exists a constant $C_{\text {obs }}>0$ such that all solutions $(\varphi, w)$ of $(3.7)$ with initial data $\left(\varphi_{0}, \varphi_{1}, w_{0}, w_{1}\right) \in H_{\text {ext }}^{1}\left(\mathcal{A}\left(r_{0}, R_{0}\right)\right) \times$ $L^{2}\left(\mathcal{A}\left(r_{0}, R_{0}\right)\right) \times\left(\mathbb{R}^{2}\right)^{2}$ satisfy

$$
\left\|\left(\varphi_{0}, \varphi_{1}, w_{0}, w_{1}\right)\right\|_{H_{\text {ext }}^{1}\left(\mathcal{A}\left(r_{0}, R_{0}\right)\right) \times L^{2}\left(\mathcal{A}\left(r_{0}, R_{0}\right)\right) \times\left(\mathbb{R}^{2}\right)^{2}}^{2} \leq C_{\text {obs }}^{2} \int_{0}^{T} \int_{\mathcal{A}\left(R_{1}, R_{0}\right)} \chi_{R_{1}<|x|<R_{0}}^{2}\left|\varphi^{\prime}(t, x)\right|^{2} \mathrm{~d} t \mathrm{~d} x .
$$

We now focus on the proof of the observability result (3.8) for the adjoint system (3.7). 


\subsection{A spherical harmonics decomposition}

In this section, we introduce polar coordinates and use a decomposition of the solutions $\varphi$ of (3.7) in spherical harmonics.

Since we are working in two dimensions, the $k$ th spherical harmonics are spanned by $\cos (k \theta), \sin (k \theta)$. Hence, we can write

$$
\begin{array}{r}
\varphi(t, x)=\frac{1}{\pi} \sum_{k=1}^{\infty}\left(\psi_{k, a}(t, r) \cos (k \theta)+\psi_{k, b}(t, r) \sin (k \theta)\right)+\frac{1}{2 \pi} \tilde{\psi}(t, r), \\
(t, x) \in(0, T) \times \mathcal{A}\left(r_{0}, R_{0}\right),
\end{array}
$$

where $x=(r, \theta)$ in the polar coordinates, $r \in\left(r_{0}, R_{0}\right), \theta \in(0,2 \pi)$.

With these notations, we have

$$
\begin{aligned}
-\Delta \varphi= & -\frac{1}{\pi} \sum_{k=1}^{\infty}\left(\left(\frac{1}{r} \partial_{r}\left(r \partial_{r} \psi_{k, a}\right)-\frac{k^{2}}{r^{2}} \psi_{k, a}\right) \cos (k \theta)\right) \\
& -\frac{1}{\pi} \sum_{k=1}^{\infty}\left(\left(\frac{1}{r} \partial_{r}\left(r \partial_{r} \psi_{k, b}\right)-\frac{k^{2}}{r^{2}} \psi_{k, b}\right) \sin (k \theta)\right)-\frac{1}{2 \pi}\left(\frac{1}{r} \partial_{r}\left(r \partial_{r} \tilde{\psi}\right)\right) .
\end{aligned}
$$

Simple computations also show that

$$
-\int_{S\left(0, r_{0}\right)} \varphi^{\prime} \boldsymbol{n} \mathrm{d} \sigma=r_{0} \int_{0}^{2 \pi} \varphi^{\prime}(t, x)\left(\begin{array}{c}
\cos (\theta) \\
\sin (\theta)
\end{array}\right) d \theta=r_{0}\left(\begin{array}{c}
\psi_{1, a}^{\prime}\left(t, r_{0}\right) \\
\psi_{1, b}^{\prime}\left(t, r_{0}\right)
\end{array}\right) .
$$

Finally, the condition $\partial_{n} \varphi(t, x)=w^{\prime}(t) \cdot \boldsymbol{n}$ on $(0, T) \times S\left(0, r_{0}\right)$ reads

$$
\partial_{r} \varphi\left(t, r_{0}, \theta\right)=w_{a}^{\prime}(t) \cos (\theta)+w_{b}^{\prime}(t) \sin (\theta),
$$

where we write

$$
w(t)=\left(\begin{array}{l}
w_{a}(t) \\
w_{b}(t)
\end{array}\right)
$$

Therefore, $(\varphi, w)$ is a solution of (3.7) if and only if the functions $\tilde{\psi}(t, r),\left(\psi_{k, a}(t, r), \psi_{k, b}(t, r)\right)_{k \geq 1}, w_{a}(t)$, $w_{b}(t)$ satisfy:

- corresponding to the zero-mode term:

$$
\left\{\begin{array}{l}
\tilde{\psi}^{\prime \prime}-\frac{1}{r} \partial_{r}\left(r \partial_{r} \tilde{\psi}\right)=0, \quad(t, r) \in(0, T) \times\left(r_{0}, R_{0}\right) \\
\partial_{r} \tilde{\psi}\left(t, r_{0}\right)=0=\tilde{\psi}\left(t, R_{0}\right), \quad t \in(0, T) \\
\tilde{\psi}(0, r)=\int_{0}^{2 \pi} \varphi_{0}(r, \theta) d \theta, r \in\left(r_{0}, R_{0}\right) \\
\tilde{\psi}^{\prime}(0, r)=\int_{0}^{2 \pi} \varphi_{1}(r, \theta) d \theta, r \in\left(r_{0}, R_{0}\right)
\end{array}\right.
$$

- corresponding to the $k$ th modes, $k \geq 2, j \in\{a, b\}$,

$$
\left\{\begin{array}{l}
\psi_{k, j}^{\prime \prime}-\frac{1}{r} \partial_{r}\left(r \partial_{r} \psi_{k, j}\right)+\frac{k^{2}}{r^{2}} \psi_{k, j}=0,(t, r) \in(0, T) \times\left(r_{0}, R_{0}\right), \\
\partial_{r} \psi_{k, j}\left(t, r_{0}\right)=0=\psi_{k, j}\left(t, R_{0}\right), \quad t \in(0, T), \\
\psi_{k, j}(0, r)=\int_{0}^{2 \pi} \varphi_{0}(r, \theta) e_{k, j}(\theta) d \theta, r \in\left(r_{0}, R_{0}\right), \\
\psi_{k, j}^{\prime}(0, r)=\int_{0}^{2 \pi} \varphi_{1}(r, \theta) e_{k, j}(\theta) d \theta, r \in\left(r_{0}, R_{0}\right),
\end{array}\right.
$$


with

$$
e_{k, j}(\theta)=\left\{\begin{array}{cc}
\cos (k \theta) & \text { if } j=a, k \geq 1 \\
\sin (k \theta) & \text { if } j=b, k \geq 1
\end{array}\right.
$$

- corresponding to the first modes, for $j \in\{a, b\}$,

$$
\begin{cases}\psi_{1, j}^{\prime \prime}-\frac{1}{r} \partial_{r}\left(r \partial_{r} \psi_{1, j}\right)+\frac{1}{r^{2}} \psi_{1, j}=0, & (t, r) \in(0, T) \times\left(r_{0}, R_{0}\right), \\ \psi_{1, j}\left(t, R_{0}\right)=0, & t \in(0, T), \\ \partial_{r} \psi_{1, j}\left(t, r_{0}\right)=\pi w_{j}^{\prime}(t), & t \in(0, T), \\ w_{j}^{\prime \prime}(t)+w_{j}(t)=r_{0} \psi_{1, j}^{\prime}\left(t, r_{0}\right), & t \in(0, T), \\ \psi_{1, j}(0, r)=\int_{0}^{2 \pi} \varphi_{0}(r, \theta) e_{1, j}(\theta) d \theta, r \in\left(r_{0}, R_{0}\right), \\ \psi_{1, j}^{\prime}(0, r)=\int_{0}^{2 \pi} \varphi_{1}(r, \theta) e_{1, j}(\theta) d \theta, r \in\left(r_{0}, R_{0}\right), \\ w_{j}(0)=w_{0} \cdot e_{j}, \quad w_{j}^{\prime}(0)=w_{1} \cdot e_{j},\end{cases}
$$

with $e_{1, j}$ as in (3.14) and

$$
e_{a}=\left(\begin{array}{l}
1 \\
0
\end{array}\right), \quad e_{b}=\left(\begin{array}{l}
0 \\
1
\end{array}\right)
$$

At this stage, the advantages of using a spherical harmonic decomposition are already clear: we have coupling with the solid only in (3.15), and not in (3.12),(3.13). Furthermore, non-locality in space in the original system (3.7) (in the source term of the equation of the oscillator) has disappeared.

For each $k \geq 2$, we define the energy $E_{k}$ corresponding to the $k$ th mode, solution of (3.13),

$$
E_{k}\left[\psi_{k}\right](t)=\int_{r_{0}}^{R_{0}}\left|\psi_{k}^{\prime}(t, r)\right|^{2} r \mathrm{~d} r+\int_{r_{0}}^{R_{0}}\left|\partial_{r} \psi_{k}(t, r)\right|^{2} r \mathrm{~d} r+\int_{r_{0}}^{R_{0}} \frac{k^{2}}{r^{2}}\left|\psi_{k}(t, r)\right|^{2} r \mathrm{~d} r .
$$

Note that the energy of solutions $\psi_{k}$ of (3.13) is constant.

We also define $E_{0}$ the energy corresponding to the 0 th harmonic:

$$
E_{0}[\tilde{\psi}](t)=\int_{r_{0}}^{R_{0}}\left|\tilde{\psi}^{\prime}(t, r)\right|^{2} r \mathrm{~d} r+\int_{r_{0}}^{R_{0}}\left|\partial_{r} \tilde{\psi}(t, r)\right|^{2} r \mathrm{~d} r
$$

which also is constant in time $t \in(0, T)$ for solutions $\tilde{\psi}$ of $(3.12)$.

Similarly, corresponding to the 1 st harmonic solutions, we introduce

$$
\begin{aligned}
\mathcal{E}_{1}\left[\psi_{1}, w\right](t)= & \int_{r_{0}}^{R_{0}}\left|\psi_{1}^{\prime}(t, r)\right|^{2} r \mathrm{~d} r+\int_{r_{0}}^{R_{0}}\left|\partial_{r} \psi_{1}(t, r)\right|^{2} r \mathrm{~d} r \\
& +\int_{r_{0}}^{R_{0}} \frac{1}{r}\left|\psi_{1}(t, r)\right|^{2} \mathrm{~d} r+\pi\left|w^{\prime}(t)\right|^{2}+\pi|w(t)|^{2},
\end{aligned}
$$

which also is independent of time for solutions $\left(\psi_{1}, w\right)$ of $(3.15)$.

Moreover, using the $L^{2}(S(0,1))$ orthogonality of the modes, the energy of solutions $(\varphi, w)$ of $(3.7)$ can be decomposed as follows

$$
E(t)=\frac{1}{4 \pi} E_{0}[\tilde{\psi}](t)+\frac{1}{2 \pi} \sum_{j \in\{a, b\}}\left(\mathcal{E}_{1}\left[\psi_{1, j}, w_{j}\right](t)+\sum_{k \geq 2} E_{k}\left[\psi_{k, j}\right](t)\right) .
$$


And similarly, the observation operator can also be diagonalized with this spherical harmonic decomposition

$$
\int_{\mathcal{A}\left(R_{1}, R_{0}\right)}\left|\varphi^{\prime}(t, x)\right|^{2} \mathrm{~d} x=\frac{1}{2 \pi} \int_{R_{1}}^{R_{0}}\left|\tilde{\psi}^{\prime}(t, r)\right|^{2} r \mathrm{~d} r+\frac{1}{\pi} \sum_{\substack{k \geq 1 \\ j \in\{a, b\}}} \int_{R_{1}}^{R_{0}}\left|\psi_{k, j}^{\prime}(t, r)\right|^{2} r \mathrm{~d} r .
$$

Therefore, system (3.7) satisfies (3.8) if and only if there exists a constant $C_{\text {obs }}$ such that

- for all $k \in \mathbb{N} \backslash\{1\}$, any solution $\psi_{k}$ of

$$
\begin{cases}\psi_{k}^{\prime \prime}-\frac{1}{r} \partial_{r}\left(r \partial_{r} \psi_{k}\right)+\frac{k^{2}}{r^{2}} \psi_{k}=0, & (t, r) \in(0, T) \times\left(r_{0}, R_{0}\right), \\ \partial_{r} \psi_{k}\left(t, r_{0}\right)=0=\psi_{k}\left(t, R_{0}\right), & t \in(0, T), \\ \psi_{k}(0, r)=\psi_{k, 0}(r) & r \in\left(r_{0}, R_{0}\right), \\ \psi_{k}^{\prime}(0, r)=\psi_{k, 1}(r) & r \in\left(r_{0}, R_{0}\right),\end{cases}
$$

satisfies

$$
E_{k}\left[\psi_{k}\right] \leq C_{\mathrm{obs}}^{2} \int_{0}^{T} \int_{R_{1}}^{R_{0}}\left|\psi_{k}^{\prime}(t, r)\right|^{2} r \mathrm{~d} r
$$

- any solution $(\psi, w)$ of

$$
\begin{cases}\psi^{\prime \prime}-\frac{1}{r} \partial_{r}\left(r \partial_{r} \psi\right)+\frac{1}{r^{2}} \psi=0, & (t, r) \in(0, T) \times\left(r_{0}, R_{0}\right), \\ \psi\left(t, R_{0}\right)=0, & t \in(0, T), \\ \partial_{r} \psi\left(t, r_{0}\right)=\pi w^{\prime}(t), & t \in(0, T), \\ w^{\prime \prime}(t)+w(t)=r_{0} \psi^{\prime}\left(t, r_{0}\right), & t \in(0, T), \\ \psi(0, r)=\psi_{0}(r) & r \in\left(r_{0}, R_{0}\right), \\ \psi^{\prime}(0, r)=\psi_{1}(r) & r \in\left(r_{0}, R_{0}\right), \\ w(0)=w_{0}, \quad w^{\prime}(0)=w_{1}, & \end{cases}
$$

satisfies

$$
\mathcal{E}_{1}[\psi, w] \leq C_{\mathrm{obs}}^{2} \int_{0}^{T} \int_{R_{1}}^{R_{0}}\left|\psi^{\prime}(t, r)\right|^{2} r \mathrm{~d} r .
$$

Note that for $k=0, \tilde{\psi}$ solves (3.20) with $k=0$ and should satisfy (3.21) with $k=0$, so that the mode $k=0$ is contained in the modes $k \in \mathbb{N} \backslash\{1\}$.

\subsection{Observability}

In the previous section, we have shown that the observability inequality (3.8) for system (3.7) is equivalent to uniform (with respect to $k$ ) observability properties (3.21) for systems (3.20) and the observability inequality (3.23) for system (3.22).

Based on that equivalence, we show the following result, which directly implies Theorem 1.2 according to Theorem 3.2:

Theorem 3.3. Let $T$ be such that

$$
T>2 \sqrt{R_{1}^{2}-r_{0}^{2}}=2 R_{1} \sqrt{1-\frac{r_{0}^{2}}{R_{1}^{2}}}
$$

Then there exists a constant $C_{\mathrm{obs}}$ such that all solutions $(\varphi, w)$ of $(3.7)$ with initial data in $H_{\mathrm{ext}}^{1}\left(\mathcal{A}\left(r_{0}, R_{0}\right)\right) \times$ $L^{2}\left(\mathcal{A}\left(r_{0}, R_{0}\right)\right) \times\left(\mathbb{R}^{2}\right)^{2}$ satisfy $(3.8)$. 
Our proof is based on the spherical harmonics decomposition performed in the previous paragraph. In particular, this indicates that we need to focus only on the observability of a family of $1 \mathrm{~d}$ wave equations (3.20) and of one non-standard wave equation (3.22) involving a coupling term with an oscillator at the boundary.

Hence, Theorem 3.3 relies on the combination of the two following propositions:

Proposition 3.4. For any time $T$ satisfying (3.24), there exists a constant $C_{\mathrm{obs}}$ independent of $k \in \mathbb{N} \backslash\{1\}$ so that for each $k \in \mathbb{N} \backslash\{1\}$, solutions of (3.20) satisfy (3.21).

Proposition 3.5. For any $T>2\left(R_{1}-r_{0}\right)$, there exists a constant $C_{\mathrm{obs}}$ such that any solution $(\psi, w)$ of $(3.22)$ satisfies (3.23).

Indeed, once these propositions are proved, according to the previous paragraph, Theorem 3.3 immediately follows, since $\sqrt{R_{1}^{2}-r_{0}^{2}} \geq R_{1}-r_{0}$. Note that the time of observability for (3.22) is not the same as for the other modes, given by (3.24). This is due to the fact that (3.22) is a one dimensional wave equation with velocity one, and then its time of observability simply is twice the length of the interval $\left(r_{0}, R_{1}\right)$, or equivalently, the width of the ring $\mathcal{A}\left(r_{0}, R_{1}\right)$.

\subsubsection{The case $k \neq 1$}

Here, we prove Proposition 3.4.

Proof of Proposition 3.4. Note that the wave equation

$$
\begin{cases}\varphi^{\prime \prime}-\Delta \varphi=0, & (t, x) \in(0, T) \times \mathcal{A}\left(r_{0}, R_{0}\right), \\ \varphi(t, x)=0, & (t, x) \in(0, T) \times S\left(0, R_{0}\right), \\ \partial_{n} \varphi(t, x)=0, & (t, x) \in(0, T) \times S\left(0, r_{0}\right), \\ \left(\varphi(0, \cdot), \varphi^{\prime}(0, \cdot)\right)=\left(\varphi_{0}, \varphi_{1}\right), & \end{cases}
$$

is observable through $\mathcal{A}\left(R_{1}, R_{0}\right)$ in any time $T$ satisfying (3.24), i.e. any solution $\varphi$ of (3.25) satisfies

$$
\int_{\mathcal{A}\left(r_{0}, R_{0}\right)}\left(\left|\nabla \varphi_{0}\right|^{2}+\left|\varphi_{1}\right|^{2}\right) \mathrm{d} x \leq C_{\text {obs }}^{2} \int_{0}^{T} \int_{\mathcal{A}\left(R_{1}, R_{0}\right)}\left|\varphi^{\prime}\right|^{2} \mathrm{~d} t \mathrm{~d} x .
$$

Indeed, the longest rays of Geometric Optics outside the control region are the ones that are tangent to $S\left(0, r_{0}\right)$ and thus this observability property is a direct consequence of the Geometric Control Condition, see [1].

Of course, using a spherical harmonic decomposition for the above problem, that yields

$$
\varphi(t, x)=\frac{1}{\pi} \sum_{k=1}^{\infty}\left(\psi_{k, a}(t, r) \cos (k \theta)+\psi_{k, b} \sin (k \theta)\right)+\frac{1}{2 \pi} \tilde{\psi}(t, r), \quad(t, x) \in(0, T) \times \mathcal{A}\left(r_{0}, R_{0}\right) .
$$

As in the previous paragraph, one easily checks that, respectively, the functions $\tilde{\psi}$ and $\left(\psi_{k, a}, \psi_{k, b}\right)_{k \geq 1}$ are solutions of (3.12) and (3.13) respectively.

Hence, one obtains that there exists a positive constant $C_{\text {obs }}$ so that

$$
\begin{aligned}
\frac{1}{2 \pi} E_{0}[\tilde{\psi}](t)+\frac{1}{\pi} \sum_{k \geq 1, j \in\{a, b\}} E_{k}\left[\psi_{k, j}\right](t) \leq & C_{\text {obs }}^{2}\left(\frac{1}{2 \pi} \int_{0}^{T^{*}} \int_{R_{1}}^{R_{0}}\left|\tilde{\psi}^{\prime}(t, r)\right|^{2} r \mathrm{~d} r \mathrm{~d} t\right. \\
& \left.+\frac{1}{\pi} \sum_{k \geq 1, j \in\{a, b\}} \int_{0}^{T^{*}} \int_{R_{1}}^{R_{0}}\left|\psi_{k, j}^{\prime}(t, r)\right|^{2} r \mathrm{~d} r \mathrm{~d} t\right),
\end{aligned}
$$

where $E_{1}$ is defined as in (3.16) for $k=1$.

Of course, estimate (3.27) implies (3.21) with the same constants $C_{\text {obs }}$ for all $k \in \mathbb{N} \backslash\{1\}$. 


\subsubsection{The case $k=1$}

This is the most intricate case, since here, we cannot rely directly on the analysis done on the classical wave equation, since the structure appears as a coupling condition on the boundary. However, due to the spherical harmonics decomposition we performed, we have reduced the problem to a 1-dimensional wave equation, for which many tools are available.

In particular, we shall use the lateral propagation of the energy, a technique that, roughly speaking, consists in switching the role of the time and space variables.

The proof of Proposition 3.5 requires the use of several lemmas and is postponed to the end of the paragraph.

To begin with, let us introduce the operator

$$
A_{1}=\left(\begin{array}{cccc}
0 & I d & 0 & 0 \\
\frac{1}{r} \partial_{r}\left(r \partial_{r} \cdot\right)-\frac{1}{r^{2}} & 0 & 0 & 0 \\
0 & 0 & 0 & I d \\
0 & r_{0} T_{r_{0}}-I d & 0
\end{array}\right), \quad T_{r_{0}}=\text { Trace Operator at } r_{0}
$$

defined in

$$
X_{1}=H_{\mathrm{ext}}^{1}\left(r_{0}, R_{0}\right) \times L^{2}\left(r_{0}, R_{0}\right) \times \mathbb{R} \times \mathbb{R}
$$

where $H_{\text {ext }}^{1}\left(r_{0}, R_{0}\right)$ is the set of functions $\psi \in H^{1}\left(r_{0}, R_{0}\right)$ satisfying $\psi\left(R_{0}\right)=0$ endowed with the norm

$$
\|\psi\|_{H_{\mathrm{ext}}^{1}\left(r_{0}, R_{0}\right)}^{2}=\int_{r_{0}}^{R_{0}} r\left|\partial_{r} \psi\right|^{2} \mathrm{~d} r
$$

with domain

$$
\mathcal{D}\left(A_{1}\right)=\left\{\left(\psi^{0}, \psi^{1}, w^{0}, w^{1}\right) \in H^{2} \cap H_{\text {ext }}^{1}\left(r_{0}, R_{0}\right) \times H_{\text {ext }}^{1}\left(r_{0}, R_{0}\right) \times \mathbb{R} \times \mathbb{R} \quad \text { and } \partial_{r} \psi^{0}\left(r_{0}\right)=\pi w^{1}\right\} .
$$

One easily checks that this operator is skew-adjoint on $X_{1}$ and with dense domain but, to justify our computations, we need a slightly stronger regularity result:

Lemma 3.6. We have

$$
\begin{aligned}
\mathcal{D}\left(A_{1}^{2}\right) & =\left\{\left(\psi^{0}, \psi^{1}, w^{0}, w^{1}\right) \in H^{3} \cap H_{\mathrm{ext}}^{1}\left(r_{0}, R_{0}\right) \times H^{2} \cap H_{\mathrm{ext}}^{1}\left(r_{0}, R_{0}\right) \times \mathbb{R} \times \mathbb{R},\right. \\
\partial_{r} \psi^{0}\left(r_{0}\right) & \left.=\pi w^{1}, \partial_{r}\left(r \partial_{r} \psi^{0}\right)\left(R_{0}\right)=0, \partial_{r} \psi^{1}\left(r_{0}\right)=\pi\left(r_{0} \psi^{1}\left(r_{0}\right)-w_{0}\right)\right\} .
\end{aligned}
$$

and $\mathcal{D}\left(A_{1}^{2}\right)$ is dense in $X_{1}$.

In particular, if $\left(\psi^{0}, \psi^{1}, w^{0}, w^{1}\right) \in \mathcal{D}\left(A_{1}^{2}\right)$, then $\psi^{0} \in C^{2}\left(\left[r_{0}, R_{0}\right]\right), \psi^{1} \in C^{1}\left(\left[r_{0}, R_{0}\right]\right)$ and the compatibility conditions

$$
\partial_{r} \psi^{0}\left(r_{0}\right)=\pi w^{1}, \quad \partial_{r}\left(r \partial_{r} \psi^{0}\right)\left(R_{0}\right)=0, \quad \partial_{r} \psi^{1}\left(r_{0}\right)=\pi\left(r_{0} \psi^{1}\left(r_{0}\right)-w_{0}\right),
$$

are satisfied.

Proof. The proof is easy and left to the reader.

We now focus on the observability property of (3.22). We first prove the following weaker version of the observability property (3.23).

Lemma 3.7. For any $T>2\left(R_{1}-r_{0}\right)$, there exists a constant $C_{\mathrm{obs}}$ such that any solution $(\psi, w)$ of $(3.22)$ satisfies

$$
\mathcal{E}_{1}[\psi, w] \leq C_{\mathrm{obs}}^{2} \int_{0}^{T} \int_{R_{1}}^{R_{0}}\left(r\left|\psi^{\prime}(t, r)\right|^{2}+r\left|\partial_{r} \psi(t, r)\right|^{2}+\frac{1}{r}|\psi(t, r)|^{2}\right) \mathrm{d} t \mathrm{~d} r .
$$


Proof. Let us first assume that $\left(\psi^{0}, \psi^{1}, w^{0}, w^{1}\right)$ satisfies

$$
\left(\psi^{0}, \psi^{1}, w^{0}, w^{1}\right) \in \mathcal{D}\left(A_{1}^{2}\right) .
$$

This regularity allows us to justify all the computations below since by Hille-Yosida theorem, the corresponding solution $(\psi, w)$ of $(3.22)$ then belongs to the functional space $C\left([0, T] ; \mathcal{D}\left(A_{1}^{2}\right)\right) \cap C^{2}\left([0, T] ; X_{1}\right)$, which guarantees a $C^{2}\left([0, T] \times\left[r_{0}, R_{0}\right]\right)$-smoothness on the function $\psi$.

Note that, by an easy density argument, it is sufficient to restrict ourselves to the proof of (3.30) for such regular data.

Let $\alpha \in\left(0, r_{0}\right)$ and introduce, for $r \in\left(r_{0}, R_{0}\right)$, the following quantity:

$$
F(r)=\int_{-r+\alpha}^{r-\alpha}\left(r\left|\psi^{\prime}(t, r)\right|^{2}+r\left|\partial_{r} \psi(t, r)\right|^{2}+\frac{1}{r}|\psi(t, r)|^{2}\right) \mathrm{d} t .
$$

Note that we are considering negative times. This can be done since system (3.22) is time-reversible.

Then

$$
\begin{aligned}
& \frac{\mathrm{d} F}{\mathrm{~d} r}(r)=\left(r\left|\psi^{\prime}(r-\alpha, r)\right|^{2}+r\left|\partial_{r} \psi(r-\alpha, r)\right|^{2}+\frac{1}{r}|\psi(r-\alpha, r)|^{2}\right) \\
& +\left(r\left|\psi^{\prime}(-r+\alpha, r)\right|^{2}+r\left|\partial_{r} \psi(-r+\alpha, r)\right|^{2}+\frac{1}{r}|\psi(-r+\alpha, r)|^{2}\right) \\
& +\int_{-r+\alpha}^{r-\alpha}\left(\left|\psi^{\prime}(t, r)\right|^{2}+\left|\partial_{r} \psi(t, r)\right|^{2}-\frac{1}{r^{2}}|\psi(t, r)|^{2}\right) \mathrm{d} t \\
& +2 \int_{-r+\alpha}^{r-\alpha}\left(r \psi^{\prime}(t, r) \partial_{r} \psi^{\prime}(t, r)+r \partial_{r} \psi(t, r) \partial_{r r} \psi(t, r)\right) \mathrm{d} t \\
& +2 \int_{-r+\alpha}^{r-\alpha}\left(\frac{1}{r} \psi(t, r) \partial_{r} \psi(t, r)\right) \mathrm{d} t
\end{aligned}
$$

so that

$$
\begin{aligned}
& \frac{\mathrm{d} F}{\mathrm{~d} r}(r)=\left(r\left|\psi^{\prime}(r-\alpha, r)\right|^{2}+r\left|\partial_{r} \psi(r-\alpha, r)\right|^{2}+\frac{1}{r}|\psi(r-\alpha, r)|^{2}\right) \\
& +\left(r\left|\psi^{\prime}(-r+\alpha, r)\right|^{2}+r\left|\partial_{r} \psi(-r+\alpha, r)\right|^{2}+\frac{1}{r}|\psi(-r+\alpha, r)|^{2}\right) \\
& +\int_{-r+\alpha}^{r-\alpha}\left(\left|\psi^{\prime}(t, r)\right|^{2}+\left|\partial_{r} \psi(t, r)\right|^{2}-\frac{1}{r^{2}}|\psi(t, r)|^{2}\right) \mathrm{d} t \\
& +2 r \psi^{\prime}(r-\alpha, r) \partial_{r} \psi(r-\alpha, r)-2 r \psi^{\prime}(-r+\alpha, r) \partial_{r} \psi(-r+\alpha, r) \\
& +2 \int_{-r+\alpha}^{r-\alpha}\left(-r \psi^{\prime \prime}(t, r) \partial_{r} \psi(t, r)+r \partial_{r} \psi(t, r) \partial_{r r} \psi(t, r)\right) \mathrm{d} t \\
& +2 \int_{-r+\alpha}^{r-\alpha}\left(\frac{1}{r} \psi(t, r) \partial_{r} \psi(t, r)\right) \mathrm{d} t \\
& \geq-\int_{-r+\alpha}^{r-\alpha}\left|\partial_{r} \psi(t, r)\right|^{2} \mathrm{~d} t-\int_{-r+\alpha}^{r-\alpha} \frac{1}{r^{2}}|\psi(t, r)|^{2} \mathrm{~d} t \\
& +\int_{-r+\alpha}^{r-\alpha} \frac{4}{r} \psi(t, r) \partial_{r} \psi(t, r) \mathrm{d} t \geq-\frac{3}{r} F(r),
\end{aligned}
$$

where we have used successively that $2 r\left|\psi^{\prime} \partial_{r} \psi\right| \leq r\left|\psi^{\prime}\right|^{2}+r\left|\partial_{r} \psi\right|^{2}$,

$$
-\psi^{\prime \prime}(t, r)+\partial_{r r} \psi(t, r)=-\frac{1}{r} \partial_{r} \psi(t, r)+\frac{1}{r^{2}} \psi(t, r),
$$


and

$$
\left|\int_{-r+\alpha}^{r-\alpha} \frac{4}{r} \psi(t, r) \partial_{r} \psi(t, r) \mathrm{d} t\right| \leq 2 \int_{-r+\alpha}^{r-\alpha}\left|\partial_{r} \psi(t, r)\right|^{2} \mathrm{~d} t+2 \int_{-r+\alpha}^{r-\alpha} \frac{1}{r^{2}}|\psi(t, r)|^{2} \mathrm{~d} t .
$$

Using (3.33), one easily gets

$$
\frac{\mathrm{d}}{\mathrm{d} r}\left(r^{3} F(r)\right) \geq 0, \quad r \in\left(r_{0}, R_{0}\right)
$$

thus yielding

$$
\forall\left(r_{a}, r_{b}\right) \in\left[r_{0}, R_{0}\right] \text { satisfying } r_{a}<r_{b}, \quad r_{a}^{3} F\left(r_{a}\right) \leq r_{b}^{3} F\left(r_{b}\right) .
$$

In particular, since $r_{0}>0$ and $R_{0}<\infty$, there exists a constant $C>0$ such that

$$
\forall\left(r_{a}, r_{b}\right) \in\left[r_{0}, R_{0}\right] \text { satisfying } r_{a}<r_{b}, \quad F\left(r_{a}\right) \leq C F\left(r_{b}\right) .
$$

Therefore,

$$
\int_{r_{0}}^{R_{1}} F(r) \mathrm{d} r \leq C F\left(R_{1}\right) \quad \text { and } \quad F\left(r_{0}\right) \leq C F\left(R_{1}\right) .
$$

Note that

$$
F\left(r_{0}\right)=\int_{-r_{0}+\alpha}^{r_{0}-\alpha}\left(r_{0}\left|\psi^{\prime}\left(t, r_{0}\right)\right|^{2}+r_{0}\left|\partial_{r} \psi\left(t, r_{0}\right)\right|^{2}+\frac{1}{r_{0}}\left|\psi\left(t, r_{0}\right)\right|^{2}\right) \mathrm{d} t .
$$

According to the transmission condition on $w^{\prime}(t)$ and $\partial_{r} \psi\left(t, r_{0}\right)$,

$$
\begin{aligned}
\int_{-r_{0}+\alpha}^{r_{0}-\alpha}\left|w^{\prime}(t)\right|^{2} \mathrm{~d} t & =\frac{1}{\pi^{2} r_{0}}\left(\int_{-r_{0}+\alpha}^{r_{0}-\alpha} r_{0}\left|\partial_{r} \psi\left(t, r_{0}\right)\right|^{2} \mathrm{~d} t\right) \\
& \leq C F\left(r_{0}\right) \leq C F\left(R_{1}\right) .
\end{aligned}
$$

Now, let $\eta=\eta(t)$ be a smooth non-negative function compactly supported in $\left(0, r_{0}-\alpha\right)$ of unit integral. Multiplying the equation of $w$ by $\eta$, we obtain

$$
\int_{0}^{r_{0}-\alpha} r_{0} \psi^{\prime}\left(t, r_{0}\right) \eta(t) \mathrm{d} t=\int_{0}^{r_{0}-\alpha} \eta(t) w(t) \mathrm{d} t-\int_{0}^{r_{0}-\alpha} \eta^{\prime}(t) w^{\prime}(t) \mathrm{d} t .
$$

Using that $w(t)=w_{0}+\int_{0}^{t} w^{\prime}(s) d s$,

$$
\begin{aligned}
\int_{0}^{r_{0}-\alpha} \eta(t) w(t) \mathrm{d} t & =w_{0}+\int_{0}^{r_{0}-\alpha}\left(\int_{0}^{t} w^{\prime}(s) d s\right) \eta(t) \mathrm{d} t \\
& =w_{0}+\int_{0}^{r_{0}-\alpha} w^{\prime}(t)\left(\int_{t}^{r_{0}-\alpha} \eta(s) d s\right) \mathrm{d} t .
\end{aligned}
$$

Therefore, for some constant $C$ depending on the choice of $\eta$ and of $r_{0}$,

$$
\begin{aligned}
\left|w_{0}\right|^{2} & \leq C\left(\int_{-r_{0}+\alpha}^{r_{0}-\alpha} r_{0}\left|\psi^{\prime}\left(t, r_{0}\right)\right|^{2} \mathrm{~d} t+\int_{-r_{0}+\alpha}^{r_{0}-\alpha}\left|w^{\prime}(t)\right|^{2} \mathrm{~d} t\right) \\
& \leq C F\left(r_{0}\right) \leq C F\left(R_{1}\right) .
\end{aligned}
$$

Estimates (3.37)-(3.38) imply

$$
\int_{-r_{0}+\alpha}^{r_{0}-\alpha}\left(\left|w^{\prime}(t)\right|^{2}+|w(t)|^{2}\right) \mathrm{d} t \leq C F\left(R_{1}\right)
$$


Therefore,

$$
\begin{aligned}
\int_{-r_{0}+\alpha}^{r_{0}-\alpha} \int_{r_{0}}^{R_{1}}\left(r\left|\psi^{\prime}(t, r)\right|^{2}+r\left|\partial_{r} \psi(t, r)\right|^{2}+\frac{1}{r}|\psi(t, r)|^{2}\right) \mathrm{d} t \mathrm{~d} r & \\
& +\int_{-r_{0}+\alpha}^{r_{0}-\alpha}\left(\pi\left|w^{\prime}(t)\right|^{2}+\pi|w(t)|^{2}\right) \mathrm{d} t \leq C F\left(R_{1}\right) .
\end{aligned}
$$

Now, let $\varepsilon>0$. Then, using (3.35), there exists a constant $C_{\varepsilon}>0$ such that

$$
F\left(R_{1}\right) \leq C_{\varepsilon} \int_{R_{1}}^{R_{1}+\varepsilon} F(r) \mathrm{d} r
$$

which implies that

$$
\begin{aligned}
& F\left(R_{1}\right) \leq C_{\varepsilon} \int_{R_{1}}^{R_{1}+\varepsilon} F(r) \mathrm{d} r \\
\leq & C_{\varepsilon} \int_{-R_{1}+\alpha-\varepsilon}^{R_{1}-\alpha+\varepsilon} \int_{R_{1}}^{R_{1}+\varepsilon}\left(r\left|\psi^{\prime}(t, r)\right|^{2}+r\left|\partial_{r} \psi(t, r)\right|^{2}+\frac{1}{r}|\psi(t, r)|^{2}\right) \mathrm{d} t \mathrm{~d} r \\
\leq & C_{\varepsilon} \int_{-R_{1}+\alpha-\varepsilon}^{R_{1}-\alpha+\varepsilon} \int_{R_{1}}^{R_{0}}\left(r\left|\psi^{\prime}(t, r)\right|^{2}+r\left|\partial_{r} \psi(t, r)\right|^{2}+\frac{1}{r}|\psi(t, r)|^{2}\right) \mathrm{d} t \mathrm{~d} r .
\end{aligned}
$$

Of course, one also has

$$
\begin{aligned}
\int_{-r_{0}+\alpha}^{r_{0}-\alpha} \int_{R_{1}}^{R_{0}}\left(r\left|\psi^{\prime}(t, r)\right|^{2}+r\left|\partial_{r} \psi(t, r)\right|^{2}\right. & \left.+\frac{1}{r}|\psi(t, r)|^{2}\right) \mathrm{d} t \mathrm{~d} r \\
& \leq \int_{-R_{1}+\alpha-\varepsilon}^{R_{1}-\alpha+\varepsilon} \int_{R_{1}}^{R_{0}}\left(r\left|\psi^{\prime}(t, r)\right|^{2}+r\left|\partial_{r} \psi(t, r)\right|^{2}+\frac{1}{r}|\psi(t, r)|^{2}\right) \mathrm{d} t \mathrm{~d} r .
\end{aligned}
$$

Hence, using (3.40)-(3.41)-(3.42), we obtain

$$
\begin{aligned}
\int_{-r_{0}+\alpha}^{r_{0}-\alpha} \int_{r_{0}}^{R_{0}}\left(r\left|\psi^{\prime}(t, r)\right|^{2}+r\left|\partial_{r} \psi(t, r)\right|^{2}\right. & \left.+\frac{1}{r}|\psi(t, r)|^{2}\right) \mathrm{d} t \mathrm{~d} r \\
& +\int_{-r_{0}+\alpha}^{r_{0}-\alpha}\left(\pi\left|w^{\prime}(t)\right|^{2}+\pi|w(t)|^{2}\right) \mathrm{d} t \\
\leq & C_{\varepsilon} \int_{-R_{1}+\alpha-\varepsilon}^{R_{1}-\alpha+\varepsilon} \int_{R_{1}}^{R_{0}}\left(r\left|\psi^{\prime}(t, r)\right|^{2}+r\left|\partial_{r} \psi(t, r)\right|^{2}+\frac{1}{r}|\psi(t, r)|^{2}\right) \mathrm{d} t \mathrm{~d} r .
\end{aligned}
$$

Of course, the left hand side of $(3.43)$ coincides with $2\left(r_{0}-\alpha\right) \mathcal{E}_{1}[\psi, w]$, since the energy $\mathcal{E}_{1}$ is constant in time for solutions of (3.22).

To sum up, we have proved that

$$
2\left(r_{0}-\alpha\right) \mathcal{E}_{1}(0) \leq C_{\varepsilon} \int_{-R_{1}+\alpha-\varepsilon}^{R_{1}-\alpha+\varepsilon} \int_{R_{1}}^{R_{0}}\left(r\left|\psi^{\prime}(t, r)\right|^{2}+r\left|\partial_{r} \psi(t, r)\right|^{2}+\frac{1}{r}|\psi(t, r)|^{2}\right) \mathrm{d} t \mathrm{~d} r,
$$

for arbitrary $\varepsilon>0$ and $\alpha \in\left(0, r_{0}\right)$.

The proof of (3.44) was done only for smooth solutions $(\psi, w)$ of $(3.22)$ but, using Lemma 3.6, a density argument immediately shows that it also holds for any solutions $(\psi, w)$ of $(3.22)$ with initial data in $H_{\text {ext }}^{1}\left(r_{0}, R_{0}\right) \times$ $L^{2}\left(r_{0}, R_{0}\right) \times \mathbb{R}^{2}$. 
Since system (3.22) is time reversible and preserves the energy $\mathcal{E}_{1}(t)$, one can do a translation in time to obtain, for all $\varepsilon>0$ and $\alpha \in\left(0, r_{0}\right)$, the existence of a positive constant $C_{\varepsilon, \alpha}$ such that all solutions of (3.22) with initial data in $H_{\mathrm{ext}}^{1}\left(r_{0}, R_{0}\right) \times L^{2}\left(r_{0}, R_{0}\right) \times \mathbb{R}^{2}$ satisfy

$$
\mathcal{E}_{1}[\psi, w] \leq C_{\varepsilon} \int_{0}^{2 R_{1}+2 \varepsilon-2 \alpha} \int_{R_{1}}^{R_{0}}\left(r\left|\psi^{\prime}(t, r)\right|^{2}+r\left|\partial_{r} \psi(t, r)\right|^{2}+\frac{1}{r}|\psi(t, r)|^{2}\right) \mathrm{d} t \mathrm{~d} r .
$$

For $T>2\left(R_{1}-r_{0}\right)$, choosing $\alpha \in\left(0, r_{0}\right)$ and $\varepsilon>0$ such that $2 R_{1}-2 \alpha+2 \varepsilon \leq T$, we obtain (3.30) for all solutions $(\psi, w)$ of $(3.22)$.

Let us now remove the term $r\left|\partial_{r} \psi\right|^{2}$ in the right hand side of (3.30):

Lemma 3.8. For any $T>2\left(R_{1}-r_{0}\right)$, there exists a constant $C_{\mathrm{obs}}$ such that any solution $(\psi, w)$ of $(3.22)$ satisfies

$$
\mathcal{E}_{1}[\psi, w] \leq C_{\mathrm{obs}}^{2} \int_{0}^{T} \int_{R_{1}}^{R_{0}}\left(r\left|\psi^{\prime}(t, r)\right|^{2}+\frac{1}{r}|\psi(t, r)|^{2}\right) \mathrm{d} t \mathrm{~d} r .
$$

Proof. Let $(\psi, w)$ be a solution of (3.22).

Let $T>2\left(R_{1}-r_{0}\right)$. Let $\varepsilon>0$ be such that $4 \varepsilon<T-2\left(R_{1}-r_{0}\right)$.

Multiplying the equation of $\psi$ by $r \eta \psi$ where $\eta=\eta(t, r)$ is nonnegative, compactly supported on $\left(R_{1}, R_{0}\right] \times$ $(0, T)$, and taking value one on $\left(R_{1}+\varepsilon, R_{0}\right) \times(\varepsilon, T-\varepsilon)$, one obtains

$$
\begin{aligned}
0= & \int_{0}^{T} \int_{r_{0}}^{R_{0}}\left(\psi^{\prime \prime}-\frac{1}{r} \partial_{r}\left(r \partial_{r} \psi\right)+\frac{1}{r^{2}} \psi\right) r \eta \psi \mathrm{d} r \mathrm{~d} t \\
= & -\int_{0}^{T} \int_{r_{0}}^{R_{0}} r \eta\left|\psi^{\prime}\right|^{2} \mathrm{~d} r \mathrm{~d} t-\int_{0}^{T} \int_{r_{0}}^{R} r \eta^{\prime} \psi^{\prime} \psi \mathrm{d} r \mathrm{~d} t \\
& +\int_{0}^{T} \int_{r_{0}}^{R_{0}} r \partial_{r} \psi \partial_{r}(\eta \psi) \mathrm{d} r \mathrm{~d} t+\int_{0}^{T} \int_{r_{0}}^{R_{0}} \frac{1}{r} \psi^{2} \eta \mathrm{d} r \mathrm{~d} t \\
= & -\int_{0}^{T} \int_{r_{0}}^{R_{0}} r \eta\left|\psi^{\prime}\right|^{2} \mathrm{~d} r \mathrm{~d} t+\frac{1}{2} \int_{0}^{T} \int_{r_{0}}^{R_{0}} r \eta^{\prime \prime}|\psi|^{2} \mathrm{~d} r \mathrm{~d} t+\int_{0}^{T} \int_{r_{0}}^{R_{0}} r \eta\left|\partial_{r} \psi\right|^{2} \mathrm{~d} r \mathrm{~d} t \\
& +\frac{1}{2} \int_{0}^{T} \int_{r_{0}}^{R_{0}} r \partial_{r}\left(\psi^{2}\right) \partial_{r} \eta \mathrm{d} r \mathrm{~d} t+\int_{0}^{T} \int_{r_{0}}^{R_{0}} \frac{1}{r} \psi^{2} \eta \mathrm{d} r \mathrm{~d} t \\
= & -\int_{0}^{T} \int_{r_{0}}^{R_{0}} r \eta\left|\psi^{\prime}\right|^{2} \mathrm{~d} r \mathrm{~d} t+\frac{1}{2} \int_{0}^{T} \int_{r_{0}}^{R_{0}} r \eta^{\prime \prime}|\psi|^{2} \mathrm{~d} r \mathrm{~d} t+\int_{0}^{T} \int_{r_{0}}^{R_{0}} r \eta\left|\partial_{r} \psi\right|^{2} \mathrm{~d} r \mathrm{~d} t \\
& -\frac{1}{2} \int_{0}^{T} \int_{r_{0}}^{R_{0}}|\psi|^{2} \partial_{r}\left(r \partial_{r} \eta\right) \mathrm{d} r \mathrm{~d} t+\int_{0}^{T} \int_{r_{0}}^{R_{0}} \frac{1}{r}|\psi|^{2} \eta \mathrm{d} r \mathrm{~d} t .
\end{aligned}
$$

Therefore,

$$
\int_{\varepsilon}^{T-\varepsilon} \int_{R_{1}+\varepsilon}^{R_{0}} r\left|\partial_{r} \psi(t, r)\right|^{2} \mathrm{~d} r \mathrm{~d} r \leq C \int_{0}^{T} \int_{R_{1}}^{R_{0}}\left(r\left|\psi^{\prime}(t, r)\right|^{2}+\frac{1}{r}|\psi(t, r)|^{2}\right) \mathrm{d} t \mathrm{~d} r .
$$

Applying Lemma 3.7 to $\tilde{T}=T-2 \varepsilon$ and $\tilde{R}_{1}=R_{1}+\varepsilon$, estimate (3.48) implies (3.46).

Let us now prove the following unique continuation result:

Lemma 3.9. For any $T>2\left(R_{1}-r_{0}\right)$, if $(\psi, w)$ is a solution of $(3.22)$ with initial data in $H_{\text {ext }}^{1}\left(r_{0}, R_{0}\right) \times$ $L^{2}\left(r_{0}, R_{0}\right) \times \mathbb{R} \times \mathbb{R}$ such that $\psi^{\prime}(t, r)$ vanishes on $(0, T) \times\left(R_{1}, R_{0}\right)$, then $(\psi, w)$ vanishes identically on $(0, T)$. 
Proof. First remark that, multiplying (3.22) by $r z^{\prime}$ for $z \in C_{c}^{\infty}\left((0, T) \times\left[r_{0}, R_{0}\right)\right)$, we get

$$
\int_{0}^{T} \int_{r_{0}}^{R_{0}} r \psi^{\prime}\left(z^{\prime \prime}-\frac{1}{r} \partial_{r}\left(r \partial_{r} z\right)+\frac{z}{r^{2}}\right) \mathrm{d} r \mathrm{~d} t=r_{0} \pi \int_{0}^{T} w^{\prime}(t) z^{\prime}\left(t, r_{0}\right) \mathrm{d} t+\int_{0}^{T}\left(w^{\prime \prime}+w\right) \partial_{r} z\left(t, r_{0}\right) \mathrm{d} t,
$$

where the last integral has to be understood in the distribution sense.

Let us first set $\varepsilon>0$ such that $2 \varepsilon<T-2\left(R_{1}-r_{0}\right)$. Then, for $f \in C_{c}^{\infty}\left(\left(R_{1}+\varepsilon-r_{0}, T-R_{1}-\varepsilon+r_{0}\right) \times\left(r_{0}, R_{0}\right)\right)$, $\eta_{0} \in C_{c}^{\infty}\left(\left(R_{1}+\varepsilon-r_{0}, T-R_{1}-\varepsilon+r_{0}\right)\right.$ and $\eta_{1} \in C_{c}^{\infty}\left(R_{1}+\varepsilon-r_{0}, T-R_{1}-\varepsilon+r_{0}\right)$, we define $\tilde{z}$ the solution of

$$
\begin{cases}\tilde{z}^{\prime \prime}-\frac{1}{r} \partial_{r}\left(r \partial_{r} \tilde{z}\right)+\frac{\tilde{z}}{r^{2}}=f, & (t, r) \in\left(-R_{0}, T+R_{0}\right) \times\left(r_{0}, R_{0}\right), \\ \tilde{z}\left(T+R_{0}, r\right)=\tilde{z}\left(-R_{0}, r\right)=0, & r \in\left(r_{0}, R_{0}\right), \\ \tilde{z}\left(t, r_{0}\right)=\eta_{0}(t), \partial_{r} \tilde{z}\left(t, r_{0}\right)=\eta_{1}(t), & t \in\left(-R_{0}, T+R_{0}\right),\end{cases}
$$

corresponding to a wave equation where we have switched the role of the time and space variables. Since it is a simple wave equation with velocity one, the solution $\tilde{z}$ is smooth, and compactly supported in the set $\left\{(t, r) \in\left(-R_{0}, T+R_{0}\right) \times\left[r_{0}, R_{0}\right]\right.$, with $\left.t \in\left(R_{1}+\varepsilon-r, T-R_{1}-\varepsilon+r\right)\right\}$.

Note that actually this can also be proved in the same spirit as the proof of Lemma 3.7 by showing that the "energy" outside the set $t \in\left(R_{1}+\varepsilon-r, T-R_{1}-\varepsilon+r\right)$ at $r$, defined by

$$
G(r)=\left(\int_{-R_{0}}^{R_{1}+\varepsilon-r}+\int_{T-R_{1}-\varepsilon+r}^{T+R_{0}}\right)\left(\left|\partial_{r} \tilde{z}(t, r)\right|^{2}+\left|\tilde{z}^{\prime}(t, r)\right|^{2}+|\tilde{z}(t, r)|^{2}\right) \mathrm{d} t,
$$

similarly as in (3.31), satisfies a differential inequality of the form $\partial_{r} G(r) \leq C G(r)$ (that can be deduced similarly as (3.33)) and the conclusion then directly comes from Gronwall's Lemma as $G\left(r_{0}\right)=0$.

Now, if we take a smooth cut-off function $\chi=\chi(r)$ taking value 1 on $\left[r_{0}, R_{1}\right]$ and vanishing on $\left[R_{1}+\varepsilon, R_{0}\right]$ for $\varepsilon>0$ and $\varepsilon<T-2\left(R_{1}-r_{0}\right), z(t, r)=\chi \tilde{z}$ is a smooth function satisfying

$$
\begin{cases}z^{\prime \prime}-\partial_{r r} z+\frac{\partial_{r} z}{r}=\chi f+\tilde{f}, & (t, r) \in\left(-R_{0}, T+R_{0}\right) \times\left(r_{0}, R_{0}\right), \\ z\left(t, r_{0}\right)=\eta_{0}(t), \partial_{r} z\left(t, r_{0}\right)=\eta_{1}(t), & t \in\left(-R_{0}, T+R_{0}\right),\end{cases}
$$

where $\tilde{f}=\left[-\partial_{r r}+\frac{\partial_{r}}{r}, \chi\right] \tilde{z}$, and $z \in C_{c}^{\infty}\left((0, T) \times\left[r_{0}, R_{0}\right)\right)$. We then apply identity (3.49) to $z$ and, using $\psi^{\prime}=0$ on $(0, T) \times\left(R_{1}, R_{0}\right)$ and the fact that $\tilde{f}$ is supported in $(0, T) \times\left(R_{1}, R_{1}+\varepsilon\right)$, we obtain

$$
\int_{0}^{T} \int_{r_{0}}^{R_{0}} \psi^{\prime} f \mathrm{~d} r \mathrm{~d} t=r_{0} \pi \int_{0}^{T} w^{\prime}(t) \eta_{0}^{\prime}(t) \mathrm{d} t+\int_{0}^{T}\left(w^{\prime \prime}+w\right) \eta_{1}(t) \mathrm{d} t,
$$

for all $f \in C_{c}^{\infty}\left(\left(R_{1}+\varepsilon-r_{0}, T-R_{1}-\varepsilon+r_{0}\right) \times\left(r_{0}, R_{0}\right)\right)$ and $\eta_{0}, \eta_{1} \in C_{c}^{\infty}\left(\left(R_{1}+\varepsilon-r_{0}, T-R_{1}-\varepsilon+r_{0}\right)\right.$. This implies that $\psi^{\prime}=0$ in $\mathcal{D}^{\prime}\left(\left(R_{1}+\varepsilon-r_{0}, T-R_{1}-\varepsilon\right) \times\left(r_{0}, R_{0}\right)\right), w^{\prime \prime}=0$ in $\mathcal{D}^{\prime}\left(\left(R_{1}+\varepsilon-r_{0}, T-R_{1}-\varepsilon\right)\right)$ and $w^{\prime \prime}+w=0$ in $\mathcal{D}^{\prime}\left(\left(R_{1}+\varepsilon-r_{0}, T-R_{1}-\varepsilon\right)\right)$. Thus $w=0$ and $w^{\prime}=0$ for $t \in\left(R_{1}+\varepsilon-r_{0}, T-R_{1}-\varepsilon\right)$.

Now, if we multiply (3.22) by $r z$ for $z \in C_{c}^{\infty}\left(\left(R_{1}+\varepsilon-r_{0}, T-R_{1}-\varepsilon\right) \times\left[r_{0}, R_{0}\right]\right)$ with $\partial_{r} z\left(t, r_{0}\right)=0$ and $z\left(t, R_{0}\right)=0$, we get

$$
0=\int_{0}^{T} \int_{r_{0}}^{R_{0}} r \psi\left(z^{\prime \prime}-\frac{1}{r} \partial_{r}\left(r \partial_{r} z\right)+\frac{z}{r^{2}}\right) \mathrm{d} r \mathrm{~d} t .
$$

Thus, for any $\eta \in C_{c}^{\infty}\left(R_{1}+\varepsilon-r_{0}, T-R_{1}-\varepsilon+r_{0}\right)$ and $\chi \in C^{\infty}\left(\left[r_{0}, R_{0}\right]\right)$ with $\partial_{r} \chi\left(r_{0}\right)=0$ and $\chi\left(R_{0}\right)=0$, taking $z=\eta \chi$, we get

$$
0=\int_{r_{0}}^{R_{0}} r\left(\int_{0}^{T} \eta \psi(t, r) \mathrm{d} t\right)\left(\chi^{\prime \prime}-\frac{1}{r} \partial_{r}\left(r \partial_{r} \chi\right)+\frac{\chi}{r^{2}}\right) \mathrm{d} r
$$


This means that for any $\eta \in C_{c}^{\infty}\left(R_{1}+\varepsilon-r_{0}, T-R_{1}-\varepsilon+r_{0}\right)$, the function $\psi_{\eta}(r)=\int_{0}^{T} \eta \psi(t, r) \mathrm{d} t$ is a weak solution of

$$
-\frac{1}{r} \partial_{r}\left(r \partial_{r} \psi_{\eta}\right)+\frac{1}{r^{2}} \psi_{\eta}=0 \text { in }\left(r_{0}, R_{0}\right), \quad \partial_{r} \psi_{\eta}\left(r_{0}\right)=\psi_{\eta}\left(R_{0}\right)=0 .
$$

Hence $\psi_{\eta}=0$ in $\mathcal{D}^{\prime}\left(r_{0}, R_{0}\right)$. Since it holds for all $\eta \in C_{c}^{\infty}\left(R_{1}+\varepsilon-r_{0}, T-R_{1}-\varepsilon+r_{0}\right)$, we have $\psi=0$ in $\mathcal{D}^{\prime}\left(\left(R_{1}+\varepsilon-r_{0}, T-R_{1}-\varepsilon\right) \times\left[r_{0}, R_{0}\right]\right)$.

This shows that $\psi, \psi^{\prime}, w, w^{\prime}$ identically vanish for $t \in\left(R_{1}+\varepsilon-r_{0}, T-R_{1}-\varepsilon\right)$. As the energy of $(\psi, w)$ is preserved in time, this concludes the proof of Lemma 3.9.

Remark 3.10. Note that the proof of the fact that under the assumptions of Lemma 3.9, $\psi^{\prime} \equiv 0$ in $\left(R_{1}-\right.$ $\left.r_{0}, T-R_{1}+r_{0}\right) \times\left(r_{0}, R_{0}\right)$ can also be derived more rapidly using Holmgren's uniqueness theorem [12]. But we have preferred to present the above proof since in this $1 \mathrm{~d}$ case this result can also be achieved using simple arguments in the spirit of the ones used in Lemma 3.7.

We are now in position to remove the extra term in (3.46) and conclude the Proof of Proposition 3.5.

Proof of Proposition 3.5. We argue by contradiction. Assume that (3.23) does not hold. Then there exists a sequence of function $\left(\psi_{0, n}, \psi_{1, n}, w_{0, n}, w_{1, n}\right)$ such that

$$
\left\|\left(\psi_{0, n}, \psi_{1, n}, w_{0, n}, w_{1, n}\right)\right\|_{H_{\mathrm{ext}}^{1}\left(r_{0}, R_{0}\right) \times L^{2}\left(r_{0}, R_{0}\right) \times \mathbb{R} \times \mathbb{R}}=1 \text { and } \lim _{n \rightarrow \infty} \int_{0}^{T} \int_{R_{1}}^{R_{0}}\left|\psi_{n}^{\prime}(t, r)\right|^{2} r \mathrm{~d} r \mathrm{~d} t=0,
$$

where $\left(\psi_{n}, w_{n}\right)$ is the corresponding solution of (3.22).

Of course, assumptions (3.51) imply that, extracting subsequence if necessary,

$$
\left(\psi_{0, n}, \psi_{1, n}, w_{0, n}, w_{1, n}\right) \rightarrow\left(\psi_{0}, \psi_{1}, w_{0}, w_{1}\right)
$$

weakly in $H_{\text {ext }}^{1}\left(r_{0}, R_{0}\right) \times L^{2}\left(r_{0}, R_{0}\right) \times \mathbb{R}^{2}$ and

$$
\int_{0}^{T} \int_{R_{1}}^{R_{0}}\left|\psi^{\prime}(t, r)\right|^{2} r \mathrm{~d} r \mathrm{~d} t=0
$$

where $(\psi, w)$ is the solution of $(3.22)$ with initial data $\left(\psi_{0}, \psi_{1}, w_{0}, w_{1}\right)$. By Lemma 3.9, (3.53) implies that $\left(\psi_{0}, \psi_{1}, w_{0}, w_{1}\right)=(0,0,0,0)$ and the convergence $(3.52)$ can be rewritten as

$$
\left(\psi_{0, n}, \psi_{1, n}, w_{0, n}, w_{1, n}\right) \rightarrow(0,0,0,0)
$$

weakly in $H_{\text {ext }}^{1}\left(r_{0}, R_{0}\right) \times L^{2}\left(r_{0}, R_{0}\right) \times \mathbb{R}^{2}$.

But (3.51) also implies that the solutions $\left(\psi_{n}, w_{n}\right)$ are uniformly bounded in $C\left([0, T] ; H_{\text {ext }}^{1}\left(r_{0}, R_{0}\right)\right) \cap$ $C^{1}\left([0, T] ; L^{2}\left(r_{0}, R_{0}\right)\right) \times C^{1}([0, T] ; \mathbb{R})$. Since $\psi_{n}$ converges in the distribution sense to 0 from $(3.54), \psi_{n}$ converges to 0 weakly $*$ in $C\left([0, T] ; H_{\text {ext }}^{1}\left(r_{0}, R_{0}\right)\right) \cap C^{1}\left([0, T] ; L^{2}\left(r_{0}, R_{0}\right)\right)$ and therefore strongly converges in $L^{2}\left((0, T) \times\left(R_{1}, R_{0}\right)\right)$ :

$$
\lim _{n \rightarrow \infty} \int_{0}^{T} \int_{R_{1}}^{R_{0}}\left|\psi_{n}(t, r)\right|^{2} r \mathrm{~d} r \mathrm{~d} t=0
$$

But the combination of (3.51) and (3.55) is not compatible with (3.46). This proves Proposition 3.5.

\subsection{Controllability statements}

We now complete the Proof of Theorem 1.2.

Proof of Theorem 1.2. Theorem 1.2 is a simple consequence of Theorems 3.2 and 3.3.

In what follows, we explain how this result can be slightly improved to more general outer boundary by combining it with the control results on the wave equation. However, the generalizations we provide will always require $\omega$ to be a disc. We shall comment about the case of $\omega$ being different from a disc in Section 4 . 


\subsubsection{More general outer boundary: when $\Omega \backslash \mathcal{C}$ is a ball}

If the set $\Omega$ is not a ball but the control set $\mathcal{C}$ is a neighborhood of $\partial \Omega$ such that $\Omega \backslash \overline{\mathcal{C}}=B\left(0, R_{1}\right)$, the result of Theorem 1.2 still holds:

Theorem 3.11. Let $\omega=B\left(0, r_{0}\right)$ as above, $\Omega$ be an open domain containing $\bar{\omega}$ and $\mathcal{C} \subset \mathcal{O}$ be a neighborhood of $\partial \Omega$ such that $\Omega \backslash \overline{\mathcal{C}}=B\left(0, R_{1}\right)$ with $R_{1}>r_{0}$. Let $T>0$ satisfying (3.24).

Then system (1.4) is null-controllable at time $T$ with controls in $L^{2}((0, T) \times \mathcal{C})$.

Sketch of the proof. We first introduce $R_{0}>R_{1}$ such that $B\left(0, R_{0}\right) \subset \Omega$. Then we truncate the solution of (1.4) in two parts similarly as in Theorem 1.1: one part corresponds to $\tilde{\Omega}=B\left(0, R_{0}\right), \omega=B\left(0, r_{0}\right)$ and the control set $\tilde{\mathcal{C}}=\mathcal{A}\left(R_{1}, R_{0}\right)$, where Theorem 1.2 applies, and the second part corresponds to $\hat{\Omega}=\Omega \backslash \overline{B\left(0, R_{1}\right)}$ with a control set $\hat{\mathcal{C}}=\hat{\Omega}=\Omega \backslash \overline{B\left(0, R_{1}\right)}$, which can be easily controlled since the control acts everywhere in the domain $\hat{\Omega}$.

We then glue these two controls similarly as in Theorem 1.1.

\subsubsection{More general outer boundary and control set $\mathcal{C}$}

Theorem 3.11 can be extended to more general control sets $\mathcal{C}$ which need not contain an entire neighborhood of the outer boundary.

In order to describe our results precisely, we shall introduce generalized bicharacteristic rays.

In order to do this, we assume that $\partial \Omega$ has no contact of infinite order with its tangent and set $M=$ $(-\infty, \infty) \times \Omega$ and $\partial M=(-\infty, \infty) \times \partial \Omega$, and $T^{*} M, T^{*} \partial M$ their respective cotangent bundles. Set then $T_{b}^{*} M=T^{*} M \cup T^{*} \partial M$ and endow it with the topology induced by the canonical projection $\pi$ of $T^{*} \bar{M}$ on $T_{b}^{*} M$. We then introduce $\Sigma_{b}=\pi\left(\left\{\rho=(t, x, \tau, \xi) \in T^{*}\left(\mathbb{R}^{3}\right)\right.\right.$, such that $\left.\left.\tau^{2}=|\xi|^{2} \neq 0\right\}\right)$, i.e. the projection of the characteristic manifold for the wave operator on $T_{b}^{*} M$.

The bicharacteristic rays are continuous curves $\gamma: \mathbb{R} \rightarrow \Sigma_{b}$ such that

- if $\gamma\left(s_{0}\right)=\left(t\left(s_{0}\right), x\left(s_{0}\right), \tau\left(s_{0}\right), \xi\left(s_{0}\right)\right) \in T^{*} M \cap \Sigma_{b}$, then $\gamma$ is differentiable at $s_{0}$ and satisfies $\frac{d \gamma}{d s}\left(s_{0}\right)=$ $H_{\square}\left(\gamma\left(s_{0}\right)\right)$, where $H_{\square}$ is the Hamiltonian vector field of the operator $\square=\partial_{t t}-\Delta$ whose principal symbol is $-\tau^{2}+|\xi|^{2}$.

- If $\gamma\left(s_{0}\right)=\left(t\left(s_{0}\right), x\left(s_{0}\right), \tau\left(s_{0}\right), \xi\left(s_{0}\right)\right) \in T^{*} \partial M \cap \Sigma_{b}$, i.e. when $x\left(s_{0}\right) \in \partial \Omega$, the behavior of the curve $\gamma$ near $s_{0}$ strongly depends on the geometry of the boundary (depending especially if $\gamma\left(s_{0}\right)$ is hyperbolic or glancing). We refer to [15] for a precise description of the broken Hamiltonian flow close to $T^{*} \partial M$.

Note that, in the above construction, we completely omit the rigid body $\omega$ within the description of the bicharacteristic rays. This is due to the fact that we do not have any precise description of the propagation of the bicharacteristic rays close to the body $\omega=B\left(0, r_{0}\right)$. Therefore, we will introduce a parameter $\varepsilon>0$ and consider only bicharacteristic rays whose projection on the physical space $s \mapsto x(s)$ stays outside $B\left(0, r_{0}+\varepsilon\right)$, at least during a certain amount of time, see Theorem 3.12 below.

Let us also remark that in our case, for $\left(t_{0}, x_{0}, \tau_{0}, \xi_{0}\right) \in T^{*} M \cap\left\{\left|\tau_{0}\right|^{2}=\left|\xi_{0}\right|^{2} \neq 0\right\}$, the bicharacteristic ray starting from $\left(t_{0}, x_{0}, \tau_{0}, \xi_{0}\right)$ simply is, locally around $s=0, \gamma: s \mapsto\left(t_{0}-2 \tau_{0} s, x_{0}+2 \xi_{0} s, \tau_{0}, \xi_{0}\right)$, which can be parametrized by the time $t$ and whose projection on the physical space simply is $t \mapsto x(t)=x_{0}-\zeta_{0} t$, where $\zeta_{0}=\xi_{0} / \tau_{0}$ is a vector of unit norm. Hence these rays travel at velocity one in $\Omega$ and correspond (in $\Omega$ ) to the classical rays of geometric optics.

Using this remark and the precise description [15] of the broken Hamiltonian flow on $T_{b}^{*} M$ for $x$ in a neighborhood of $\partial \Omega$, the bicharacteristic rays are actually defined up to re-parametrization on $S \Sigma=\Sigma_{b} / \sim$ where the quotient is taken with respect to the relation $\rho_{1}=\left(t_{1}, x_{1}, \tau_{1}, \xi_{1}\right) \sim \rho_{2}=\left(t_{2}, x_{2}, \tau_{2}, \xi_{2}\right)$ if $\left(t_{1}, x_{1}\right)=\left(t_{2}, x_{2}\right)$ and there exists $\alpha \in \mathbb{R}_{+}^{*}$ such that $\left(\tau_{1}, \xi_{1}\right)=\alpha\left(\tau_{2}, \xi_{2}\right)$. Indeed, if two bicharacteristic rays $\gamma_{1}, \gamma_{2}$ are in the same equivalence class for some $s_{1}, s_{2}$, i.e. $\gamma_{1}\left(s_{1}\right) \sim \gamma_{2}\left(s_{2}\right)$ which means in particular $\left(\tau_{1}, \xi_{1}\right)=\alpha\left(\tau_{2}, \xi_{2}\right)$ for some $\alpha>0$, then for all $s \in \mathbb{R}, \gamma_{1}(s)=\gamma_{2}(g(s))$, where $g(s)=s_{2}+\alpha\left(s-s_{1}\right)$. In the following, all bicharacteristic rays may simply be parametrized by the time $t$ by imposing the additional constraint $\left|\tau_{0}\right|=1 / 2$ as 


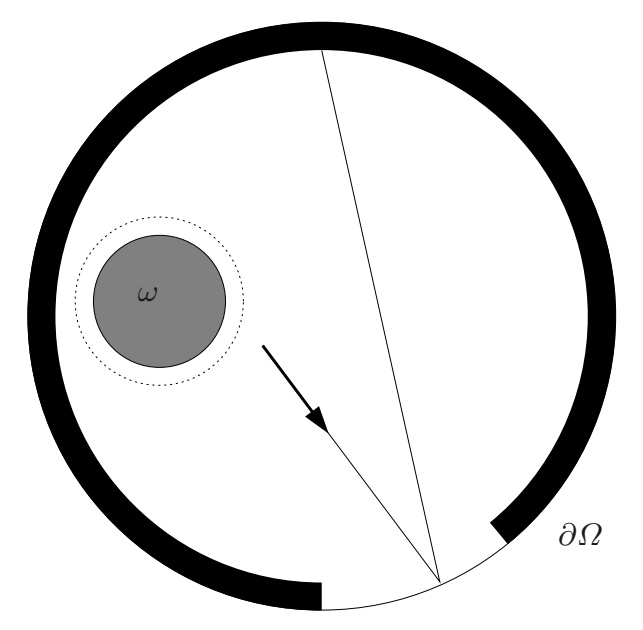

FiguRE 4. A geometric setting which satisfies the geometric control condition of Theorem 3.12: $\mathcal{C}$ is the set in black. A disk $B\left(0, r_{0}+\varepsilon\right)$ satisfying the assumptions is drawn in dot lines. The $x$-projection of a generalized bicharacteristic ray is drawn.

$t \mapsto \gamma(t)=(t, x(t), \tau(t), \xi(t)) \in \Sigma_{b}$ if $\tau_{0}=-1 / 2$, and as $t \mapsto \gamma(t)=(-t, x(t), \tau(t), \xi(t)) \in \Sigma_{b}$ if $\tau_{0}=1 / 2$. (Note that, along these bicharacteristic ray, $t \mapsto \tau(t)$ is constant and $\left.\left|\xi_{0}\right|=1 / 2\right)$.

Note that the above identification preserves the orientation of the bicharacteristic rays. But, if we do not care about the orientation of the rays, remark then that for any $\gamma_{0}=\left(t_{0}, x_{0}, \tau_{0}, \xi_{0}\right) \in T_{b}^{*} M \cap \Sigma_{b}$ with $t_{0}=0$, $\tau_{0}=1 / 2=\left|\xi_{0}\right|$, denoting $t \mapsto \gamma(t)=(t, x(t), \tau(t), \xi(t))$ the bicharacteristic ray starting from $\gamma_{0}$, the set $\{(t, x(t)), t \in \mathbb{R}\}$ coincide with the set $\{(t, \tilde{x}(t)), t \in \mathbb{R}\}$, where $\tilde{\gamma}: t \mapsto \tilde{\gamma}(t)=(t, \tilde{x}(t), \tau(t), \xi(t))$ is the bicharacteristic ray starting from $\left(0, x_{0},-\tau_{0},-\xi_{0}\right)$, the only difference between $\gamma$ and $\tilde{\gamma}$ being their orientation: $\gamma$ is parametrized forward in time while $\tilde{\gamma}$ is parametrized backward in time.

We are then in position to state the following result:

Theorem 3.12. Let $\omega=B\left(0, r_{0}\right), \Omega$ be a smooth open domain such that $\bar{\omega} \subset \Omega$ and assume that $\mathcal{C}$ is an open subset of $\mathcal{O}=\Omega \backslash \overline{B\left(0, r_{0}\right)}$ such that for some $\varepsilon>0$,

(H1) $\overline{B\left(0, r_{0}+\varepsilon\right)} \subset \Omega$ and $\mathcal{C} \subset \Omega \backslash \overline{B\left(0, r_{0}+\varepsilon\right)}$;

(H2) $\partial \Omega$ has no contact of infinite order with its tangents.

(H3) There exists $T_{0}^{\varepsilon}>0$ such that the following geometric condition is satisfied:

For all generalized bicharacteristic rays $\gamma: \mathbb{R} \rightarrow \Sigma_{b}, t \mapsto \gamma(t)=(t, x(t), \tau(t), \xi(t))$ starting from $\left(t_{0}, x_{0}, \tau_{0}, \xi_{0}\right) \in T_{b}^{*} M \cap \Sigma_{b}$ with $t_{0}=0, x_{0} \notin \overline{B\left(0, r_{0}+\varepsilon\right)}$ and $-\tau_{0}=\left|\xi_{0}\right|=1 / 2$, there exists a neighborhood $\left(-T_{-}, T_{+}\right) \subset\left(-T_{0}^{\varepsilon}, T_{0}^{\varepsilon}\right)$ of 0 such that for all $t \in\left(-T_{-}, T_{+}\right), x(t) \in \bar{\Omega} \backslash \overline{B\left(0, r_{0}+\varepsilon\right)}$ and $\mathcal{C} \cap\left\{x(t), t \in\left(-T_{-}, T_{+}\right)\right\} \neq \emptyset$.

Then system (1.4) is null-controllable with controls in $L^{2}((0, T) \times \mathcal{C})$ in any time $T$ satisfying $T>2 T_{0}^{\varepsilon}+$ $2 \sqrt{\left(r_{0}+\varepsilon\right)^{2}-r_{0}^{2}}$.

Condition (H3) can be interpreted as follows: for each generalized bicharacteristic, there is a direction of time $t$ such that the bicharacteristic ray meets $\mathcal{C}$ before meeting $\overline{B\left(0, r_{0}+\varepsilon\right)}$ and in a time interval of length $<T_{0}^{\varepsilon}$.

Figure 4 provides an example satisfying the geometric conditions of Theorem 3.12. Figure 4 also presents a ray of geometric optics for which only one direction of time meets the control region without entering the ball $B\left(0, r_{0}+\varepsilon\right)$. 
Proof. Let $\varepsilon>0$ be such that $\overline{B\left(0, r_{0}+\varepsilon\right)} \subset \Omega$ and $\mathcal{C} \subset \Omega \backslash \overline{B\left(0, r_{0}+\varepsilon\right)}$. From Theorem 3.11, we have a controllability result, hence an observability result according to [13], from the set $\Omega \backslash \overline{B\left(0, r_{0}+\varepsilon\right)}$ : for any $T_{\varepsilon}>2 \sqrt{\left(r_{0}+\varepsilon\right)^{2}-r_{0}^{2}}$, there exists a constant $C_{\mathrm{obs}, \varepsilon}>0$ such that

$$
\left\|\left(\varphi_{0}, \varphi_{1}, w_{0}, w_{1}\right)\right\|_{H_{\mathrm{ext}}^{1}(\mathcal{O}) \times L^{2}(\mathcal{O}) \times\left(\mathbb{R}^{2}\right)^{2}}^{2} \leq C_{\mathrm{obs}, \varepsilon}^{2} \int_{0}^{T_{\varepsilon}} \int_{\Omega \backslash \overline{B\left(0, r_{0}+\varepsilon\right)}} \chi_{|x|>r_{0}+\varepsilon}^{2}\left|\varphi^{\prime}(t, x)\right|^{2} \mathrm{~d} t \mathrm{~d} x,
$$

where $(\varphi, w)$ is the solution of

$$
\begin{cases}\varphi^{\prime \prime}-\Delta \varphi=0, & (t, x) \in(0, T) \times \mathcal{O}, \\ \varphi(t, x)=0, & (t, x) \in(0, T) \times \partial \Omega, \\ \partial_{n} \varphi(t, x)=w^{\prime} \cdot \boldsymbol{n}, & (t, x) \in(0, T) \times S\left(0, r_{0}\right), \\ w^{\prime \prime}+w=-\int_{S\left(0, r_{0}\right)} \varphi^{\prime} \boldsymbol{n} \mathrm{d} \sigma, & t \in(0, T), \\ \left(\varphi(0, \cdot), \varphi^{\prime}(0, \cdot)\right)=\left(\varphi_{0}, \varphi_{1}\right), & \\ \left(w(0), w^{\prime}(0)\right)=\left(w_{0}, w_{1}\right) . & \end{cases}
$$

Assume now that the control set $\mathcal{C}$ satisfies the geometric Condition (H3) of Theorem 3.12 in time $T_{0}^{\varepsilon}$.

Under this assumption, using the results of propagation of singularity for the waves $([3,4])$, a contradiction argument based on (3.56) yields that any solution $(\varphi, w)$ of $(3.57)$ satisfies, for any $T>2 T_{0}^{\varepsilon}+2 \sqrt{\left(r_{0}+\varepsilon\right)^{2}-r_{0}^{2}}$,

$$
\left\|\left(\varphi_{0}, \varphi_{1}, w_{0}, w_{1}\right)\right\|_{H_{\text {ext }}^{1}(\mathcal{O}) \times L^{2}(\mathcal{O}) \times\left(\mathbb{R}^{2}\right)^{2}}^{2} \leq C_{\text {obs }}^{2} \int_{0}^{T} \int_{\mathcal{C}} \chi_{\mathcal{C}}^{2}\left|\varphi^{\prime}(t, x)\right|^{2} \mathrm{~d} t \mathrm{~d} x .
$$

Indeed, assume that (3.58) is false. Then there exists a sequence $\left(\varphi^{n}, w^{n}\right)$ of solutions of $(3.57)$, with initial data $\left(\varphi_{0}^{n}, \varphi_{1}^{n}, w_{0}^{n}, w_{1}^{n}\right)$ of unit norm in $H_{\text {ext }}^{1}(\mathcal{O}) \times L^{2}(\mathcal{O}) \times\left(\mathbb{R}^{2}\right)^{2}$ for which $\left(\varphi^{n}\right)^{\prime}$ converges to 0 strongly in $L^{2}((0, T) \times \mathcal{C})$. Of course, extracting sequences if needed, $\left(\varphi_{0}^{n}, \varphi_{1}^{n}, w_{0}^{n}, w_{1}^{n}\right)$ weakly converges to $\left(\Phi_{0}, \Phi_{1}, W_{0}, W_{1}\right)$ in $H_{\text {ext }}^{1}(\mathcal{O}) \times L^{2}(\mathcal{O}) \times\left(\mathbb{R}^{2}\right)^{2}$, and the corresponding solution $(\Phi, W)$ to $(3.57)$ is such that $\Phi^{\prime}$ vanishes identically on $(0, T) \times \mathcal{C}$.

Using Holmgren's uniqueness theorem [12] (see also [13], Chap. 1, Sect. 8 for more precisions), one thus gets $\Phi^{\prime}=0$ on $\left(T_{0}^{\varepsilon}, T-T_{0}^{\varepsilon}\right) \times\left(\Omega \backslash \overline{B\left(0, r_{0}+\varepsilon\right)}\right)$. At this stage, let us emphasize that Holmgren's uniqueness theorem is a local argument of "propagation of zero": it can thus be applied in the set $\{(t, x) \in(0, T) \times$ $\Omega \backslash \overline{B\left(0, r_{0}+\varepsilon\right)}$, with $\left.d(x, \mathcal{C})+|t-T / 2| \leq T / 2\right\}$, where the distance is the geodesic distance on $\Omega \backslash \overline{B\left(0, r_{0}+\varepsilon\right)}$. This set contains $\left(T_{0}^{\varepsilon}, T-T_{0}^{\varepsilon}\right) \times\left(\Omega \backslash \overline{B\left(0, r_{0}+\varepsilon\right)}\right)$ according to assumption (H3).

Remark then that, since (3.57) is autonomous, that is time-translation invariant, the observability estimate (3.56) is valid over any interval of observability of length $>2 \sqrt{\left(r_{0}+\varepsilon\right)^{2}-r_{0}^{2}}$. Thus, applying (3.56), $\Phi$ and $W$ vanishes identically everywhere.

Hence the sequence $\left(\varphi^{n}, w^{n}\right)$ weakly converges to $(0,0)$ in $H^{1}((0, T) \times \mathcal{O}) \times H^{1}\left(0, T ; \mathbb{R}^{2}\right)$ and $\left(\varphi^{n}\right)^{\prime}$ strongly converges to 0 in $L^{2}((0, T) \times \mathcal{C})$. Hence the micro-local defect measures $\mu=\mu(t, x, \tau, \xi)$ of the sequence $\left(\varphi^{n}\right)^{\prime}$ vanishes identically on $(0, T) \times \mathcal{C} \times\left(\left(\mathbb{R}^{3} \backslash\{0\}\right) \cap\{|\tau|=|\xi|=1 / 2\}\right)$. According to the propagation of the singularities for the waves $[3,4,11], \mu$ is constant along the bicharacteristics whose projection in the physical space stays outside $B\left(0, r_{0}+\varepsilon\right)$, and thus $\mu$ identically vanishes for $(t, x) \in\left(T_{0}^{\varepsilon}, T-T_{0}^{\varepsilon}\right) \times\left(\Omega \backslash \overline{B\left(0, r_{0}+\varepsilon\right)}\right)$. This implies that $\left(\varphi^{n}\right)^{\prime}$ strongly converges to 0 on $\left(T_{0}^{\varepsilon}, T-T_{0}^{\varepsilon}\right) \times\left(\Omega \backslash \overline{B\left(0, r_{0}+\varepsilon\right)}\right)$, which contradicts (3.56) and the fact that $\left(\varphi_{0}^{n}, \varphi_{1}^{n}, w_{0}^{n}, w_{1}^{n}\right)$ is of unit norm in $H_{\text {ext }}^{1}(\mathcal{O}) \times L^{2}(\mathcal{O}) \times\left(\mathbb{R}^{2}\right)^{2}$.

One then immediately deduce Theorem 3.12 from (3.58) and Theorem 3.2.

\subsection{Boundary controllability}

Let us now briefly explain what can be said about the case of an observation/control from the external boundary $S\left(0, R_{0}\right)$, still in the radial case. Again, the crucial step of our results is an observability result, similar to Theorem 3.3 . 
Indeed, the Proof of Theorem 3.3 can be modified easily to deal with an observation on the external boundary:

Theorem 3.13. Let $r_{0}<R_{0}$ and set $\omega=B\left(0, r_{0}\right), \mathcal{O}=\mathcal{A}\left(r_{0}, R_{0}\right)$.

Let $T$ be such that

$$
T>2 \sqrt{R_{0}^{2}-r_{0}^{2}}
$$

Then there exists a constant $C_{\mathrm{obs}}$ such that all solutions $(\varphi, w)$ of $(3.7)$ with initial data in $H_{\mathrm{ext}}^{1}\left(\mathcal{A}\left(r_{0}, R_{0}\right)\right) \times$ $L^{2}\left(\mathcal{A}\left(r_{0}, R_{0}\right)\right) \times\left(\mathbb{R}^{2}\right)^{2}$ satisfy

$$
\left\|\left(\varphi_{0}, \varphi_{1}, w_{0}, w_{1}\right)\right\|_{H_{\text {ext }}^{1}\left(\mathcal{A}\left(r_{0}, R_{0}\right)\right) \times L^{2}\left(\mathcal{A}\left(r_{0}, R_{0}\right)\right) \times\left(\mathbb{R}^{2}\right)^{2}}^{2} \leq C_{\text {obs }}^{2} \int_{0}^{T} \int_{S\left(0, R_{0}\right)}\left|\partial_{n} \varphi(t, x)\right|^{2} \mathrm{~d} t \mathrm{~d} \sigma .
$$

Sketch of the proof. The proof is similar to that of Theorem 3.3: it relies on the spherical harmonics decomposition to allow to consider only the boundary observability of the equations (3.20) and (3.22).

Of course, still using [1], Proposition 3.4 can be replaced by the following one:

Proposition 3.14. For any time $T$ satisfying (3.59), there exists a constant $C_{\mathrm{obs}}$ so that for all $k \in \mathbb{N} \backslash\{1\}$, solutions $\psi_{k}$ of (3.20) satisfy

$$
E_{k}\left[\psi_{k}\right] \leq C_{\mathrm{obs}}^{2} \int_{0}^{T} R_{0}\left|\partial_{r} \psi_{k}\left(t, R_{0}\right)\right|^{2} \mathrm{~d} t
$$

And again, using the lateral propagation of the energy, one derives:

Proposition 3.15. For any $T>2\left(R_{0}-r_{0}\right)$, there exists a constant $C_{\mathrm{obs}}$ such that any solution $(\psi, w)$ of (3.22) satisfies

$$
\mathcal{E}_{1}[\psi, w] \leq C_{\text {obs }}^{2} \int_{0}^{T} R_{0}\left|\partial_{r} \psi_{1}\left(t, R_{0}\right)\right|^{2} \mathrm{~d} t .
$$

Actually, the proof of Proposition 3.15 is even simpler than that of Proposition 3.5 since it reduces to the lateral propagation of the energy argument developed in Lemma 3.7. This follows from the fact that

$$
F\left(R_{0}\right)=\int_{-R_{0}+\alpha}^{R_{0}-\alpha} R\left|\partial_{r} \psi\left(t, R_{0}\right)\right|^{2} \mathrm{~d} t
$$

precisely coincides with the observed quantity and then (3.40) with $R_{1}=R_{0}$ immediately yields (3.62). In particular, Lemmas 3.8 and 3.9 are not needed in that case of boundary observation.

Once observability is established, using the Hilbert Uniqueness Method introduced in [13], one can prove a boundary controllability result similar to Theorem 1.2 .

But before stating this result, one should explain what is the functional setting for the Cauchy problem associated to non-homogeneous boundary conditions on the outer boundary $\partial \Omega$ :

Theorem $3.16([18])$. Let $H_{\text {ext }}^{1}(\mathcal{O})^{*}$ be the dual of $H_{\text {ext }}^{1}(\mathcal{O})$. Define $Y$ as the completion of $H_{\text {ext }}^{1}(\mathcal{O}) \times L^{2}(\mathcal{O}) \times$ $\left(\mathbb{R}^{2}\right)^{2}$ with respect to the norm

$$
\left\|\left(y_{0}, y_{1}, s_{0}, s_{1}\right)\right\|_{Y}^{2}=\left\|y_{0}\right\|_{L^{2}(\mathcal{O})}^{2}+\left\|y_{1}\right\|_{H_{\text {ext }}^{1}(\mathcal{O})^{*}}^{2}+\left|s_{0}\right|^{2}+\left|s_{1}+\int_{\partial \omega} y_{0} \boldsymbol{n} \mathrm{d} \sigma\right|^{2} .
$$

For any $\left(y_{0}, y_{1}, s_{0}, s_{1}\right) \in Y$ and $f \in L^{2}((0, T) \times \partial \Omega)$, there exists a unique solution $(y, s)$ of

$$
\begin{cases}y^{\prime \prime}-\Delta y=0, & (t, x) \in(0, T) \times \mathcal{O}, \\ y(t, x)=f, & (t, x) \in(0, T) \times \partial \Omega, \\ \partial_{n} y(t, x)=s^{\prime} \cdot \boldsymbol{n}, & (t, x) \in(0, T) \times \partial \omega, \\ s^{\prime \prime}+s=-\int_{\partial \omega} y^{\prime} \boldsymbol{n} \mathrm{d} \sigma, & t \in(0, T), \\ \left(y(0, \cdot), y^{\prime}(0, \cdot)\right)=\left(y_{0}, y_{1}\right), & \\ \left(s(0), s^{\prime}(0)\right)=\left(s_{0}, s_{1}\right) . & \end{cases}
$$

in the transposition sense satisfying $\left(y, y^{\prime}, s, s^{\prime}\right) \in C([0, T] ; Y)$. 
Besides, there exists a constant $C$ such that

$$
\sup _{t \in[0, T]}\left\|\left(y(t), y^{\prime}(t), s(t), s^{\prime}(t)\right)\right\|_{Y} \leq C\left(\|f\|_{L^{2}((0, T) \times \partial \Omega)}+\left\|\left(y_{0}, y_{1}, s_{0}, s_{1}\right)\right\|_{Y}\right) .
$$

The proof is done by duality as in the context of the non-homogeneous wave equation (see [13]). It is then based on hidden regularity results that are easy to adapt from the ones obtained for the classical waves in [13]. To be more precise, these results state that, given a solution of the wave equation in the energy space $H_{0}^{1}(\mathcal{O}) \times L^{2}(\mathcal{O})$, the normal derivative on the boundary lies in $L^{2}((0, T) \times \mathcal{O})$ (note that this is not a consequence of the classical Sobolev embeddings). Here, there is an additional difficulty which consists in identifying the dual of the energy space $H_{\text {ext }}^{1}(\mathcal{O}) \times L^{2}(\mathcal{O}) \times\left(\mathbb{R}^{2}\right)^{2}$ when $L^{2}(\mathcal{O})$ is identified with its dual. This difficulty has been raised and solved in [18] where it is shown that it can be identified with the space $Y$ defined in Theorem 3.16.

We now state the following counterpart of Theorem 3.2:

Theorem 3.17. Assume that $T>0$ is such that the observability property

$$
\left\|\left(\varphi_{0}, \varphi_{1}, w_{0}, w_{1}\right)\right\|_{H_{\mathrm{ext}}^{1}(\mathcal{O}) \times L^{2}(\mathcal{O}) \times\left(\mathbb{R}^{2}\right)^{2}}^{2} \leq C_{\mathrm{obs}}^{2} \int_{0}^{T} \int_{\partial \Omega}\left|\partial_{n} \varphi(t, x)\right|^{2} \mathrm{~d} t \mathrm{~d} \sigma
$$

holds for all solutions of (1.12).

Then, given any initial data $\left(y_{0}, y_{1}, s_{0}, s_{1}\right) \in Y$, there exists a control function $f \in L^{2}((0, T) \times \partial \Omega)$ such that the corresponding solution $(y, s)$ of (3.64) satisfies the null-controllability requirement (1.5).

Besides, it can be computed as follows. Let us define the functional $J$ on $H_{\text {ext }}^{1}(\mathcal{O}) \times L^{2}(\mathcal{O}) \times\left(\mathbb{R}^{2}\right)^{2}$ by

$$
\begin{aligned}
& J\left(\varphi_{0}, \varphi_{1}, w_{0}, w_{1}\right)=\frac{1}{2} \int_{0}^{T} \int_{\partial \Omega}\left|\partial_{n} \varphi(t, x)\right|^{2} \mathrm{~d} t \mathrm{~d} \sigma \\
& -\left\langle y_{1}, \varphi_{0}\right\rangle_{H_{\mathrm{ext}}^{1}(\mathcal{O})^{*}, H_{\mathrm{ext}}^{1}(\mathcal{O})}+\int_{\mathcal{O}} \varphi_{1} y_{0} \\
& +s_{0} \cdot\left(\int_{\partial \omega} \varphi_{0} \boldsymbol{n} d \sigma+w_{1}\right)-w_{0} \cdot\left(s_{1}+\int_{\omega} y_{0} \boldsymbol{n} \mathrm{d} \sigma\right) .
\end{aligned}
$$

The functional $J$ has a unique minimizer $\left(\tilde{\varphi}_{0}, \tilde{\varphi}_{1}, \tilde{w}_{0}, \tilde{w}_{1}\right)$ in $H_{\mathrm{ext}}^{1}(\mathcal{O}) \times L^{2}(\mathcal{O}) \times\left(\mathbb{R}^{2}\right)^{2}$. Denoting by $(\tilde{\varphi}, \tilde{w})$ the corresponding solution of (3.7), the function

$$
f(t, x)=\partial_{n} \tilde{\varphi}(t, x), \quad(t, x) \in(0, T) \times \partial \Omega,
$$

is an admissible control function for (3.64).

Moreover, it has minimal $L^{2}((0, T) \times \partial \Omega)$-norm among all admissible controls for (3.64).

Such Theorem is classical and we refer to [13] for its proof, which can also be done similarly as Theorem 3.2.

Combining Theorems 3.17 with the observability results stated in Theorem 3.13, we obtain:

Theorem 3.18. Let $r_{0}<R_{0}$ and set $\omega=B\left(0, r_{0}\right), \mathcal{O}=\mathcal{A}\left(r_{0}, R_{0}\right), \Omega=B\left(0, R_{0}\right)$. Let $T>0$ satisfying (3.59). Then system (3.64) is exactly controllable at time $T$ through the outer boundary $\partial \Omega=S\left(0, R_{0}\right)$, that is: given any initial data $\left(y_{0}, y_{1}, s_{0}, s_{1}\right) \in Y$, there exists a control function $f \in L^{2}((0, T) \times \partial \Omega)$ such that the corresponding solution $(y, s)$ of (3.64) satisfies the null-controllability requirement (1.5).

Of course, similarly as in the distributed case handled in Section 3.4, one can consider more general outer boundaries using the propagation of singularity derived in $[2,4]$. Statements are left to the reader. 


\subsection{Homogeneous Neumann boundary conditions on the external boundary}

The model under consideration is also relevant with Neumann boundary conditions $\partial_{n} y=0$ on the external boundary $\partial \Omega$. In this case, when the control set $\mathcal{C}$ is a neighborhood of the whole external boundary $\partial \Omega$, one can easily derive the same control results as in the case of Dirichlet boundary conditions by a simple gluing argument in the spirit of Theorem 3.11: one would then obtain control results with control in $L^{2}((0, T) \times \mathcal{C})$ for data in $H^{1}(\mathcal{O}) \times L^{2}(\mathcal{O}) \times\left(\mathbb{R}^{2}\right)^{2}$ under the same conditions on the time and on the control set. But to handle situations where the control set $\mathcal{C}$ does not necessarily contain an entire neighborhood of $\partial \Omega$, one should be careful in the duality between observability and controllability and explain the counterpart of Theorem 3.2 in this case.

We suggest using the following duality argument: $f \in L^{2}((0, T) \times \mathcal{C})$ is a control for $\left(y_{0}, y_{1}, s_{0}, s_{1}\right) \in H^{1}(\mathcal{O}) \times$ $L^{2}(\mathcal{O}) \times\left(\mathbb{R}^{2}\right)^{2}$, that is the solution $(y, s)$ of

$$
\begin{cases}y^{\prime \prime}-\Delta y=f \chi_{\mathcal{C}}, & (t, x) \in(0, T) \times \mathcal{O}, \\ \partial_{n} y(t, x)=0, & (t, x) \in(0, T) \times \partial \Omega, \\ \partial_{n} y(t, x)=s^{\prime} \cdot \boldsymbol{n}, & (t, x) \in(0, T) \times \partial \omega, \\ s^{\prime \prime}+s=-\int_{\partial \omega} y^{\prime} \boldsymbol{n} \mathrm{d} \sigma, & t \in(0, T), \\ \left(y(0, \cdot), y^{\prime}(0, \cdot)\right)=\left(y_{0}, y_{1}\right), & \\ \left(s(0), s^{\prime}(0)\right)=\left(s_{0}, s_{1}\right), & \end{cases}
$$

satisfies (1.5), if and only if for all $\left(\varphi_{0}, \varphi_{1}, w_{0}, w_{1}\right) \in H^{1}(\mathcal{O}) \times L^{2}(\mathcal{O}) \times\left(\mathbb{R}^{2}\right)^{2}$ and $m \in \mathbb{R}$, the solution $(\varphi, w)$ of

$$
\begin{cases}\varphi^{\prime \prime}-\Delta \varphi=-m, & (t, x) \in(0, T) \times \mathcal{O}, \\ \partial_{n} \varphi(t, x)=0, & (t, x) \in(0, T) \times \partial \Omega, \\ \partial_{n} \varphi(t, x)=w^{\prime} \cdot \boldsymbol{n}, & (t, x) \in(0, T) \times \partial \omega, \\ w^{\prime \prime}+w=-\int_{\partial \omega} \varphi^{\prime} \boldsymbol{n} \mathrm{d} \sigma, & t \in(0, T), \\ \left(\varphi(0, \cdot), \varphi^{\prime}(0, \cdot)\right)=\left(\varphi_{0}, \varphi_{1}\right), & \\ \left(w(0), w^{\prime}(0)\right)=\left(w_{0}, w_{1}\right) . & \end{cases}
$$

satisfies

$$
0=\int_{0}^{T} \int_{\mathcal{C}} \chi_{\mathcal{C}} f \varphi^{\prime}+m \int_{\mathcal{O}} y_{0}+\int_{\mathcal{O}} \nabla \varphi_{0} \cdot \nabla y_{0}+\int_{\mathcal{O}} \varphi_{1} y_{1}+\left(s_{0} \cdot w_{0}+s_{1} \cdot w_{1}\right) .
$$

Of course, this equivalence is the counterpart of the necessary and sufficient condition (3.6). Note however that we have introduced a source term $m$ in the adjoint equation (3.69). This is due to the fact that $\left\|\nabla y_{0}\right\|_{L^{2}}$ is not a norm on $H^{1}(\mathcal{O})$ and we shall instead propose the norm $\left\|\nabla y_{0}\right\|_{L^{2}}+\left|\int_{\mathcal{O}} y_{0}\right|$. Also remark that replacing $\varphi_{0}$ by $\varphi_{0}+c$ for constants $c \in \mathbb{R}$, the solution of (3.69) is modified into $\varphi+c$ instead of $\varphi$, but $\varphi^{\prime}$ is not modified by a constant shift on $\varphi_{0}$. Therefore solutions $(\varphi, w)$ of $(3.69)$ are given in $C\left([0, T] ; H^{1}(\mathcal{O}) / \mathbb{R}\right) \cap C^{1}\left([0, T] ; L^{2}(\mathbb{R})\right) \times C^{1}\left([0, T] ; \mathbb{R}^{2}\right)$ for initial data $\left(\varphi_{0}, \varphi_{1}, s_{0}, s_{1}\right) \in H^{1}(\mathcal{O}) / \mathbb{R} \times L^{2}(\mathbb{R}) \times\left(\mathbb{R}^{2}\right)^{2}$ and source term $m \in \mathbb{R}$, where $H^{1}(\mathcal{O}) / \mathbb{R}$ is the space $H^{1}(\mathcal{O})$ quotiented by constants.

Hence, $f \in L^{2}((0, T) \times \mathcal{C})$ is a control for $\left(y_{0}, y_{1}, s_{0}, s_{1}\right) \in H^{1}(\mathcal{O}) \times L^{2}(\mathcal{O}) \times\left(\mathbb{R}^{2}\right)^{2}$ if and only if (3.70) holds for all solutions $(\varphi, w)$ of $(3.69)$ with initial data $\left(\varphi_{0}, \varphi_{1}, w_{0}, w_{1}\right) \in H^{1}(\mathcal{O}) / \mathbb{R} \times L^{2}(\mathcal{O}) \times\left(\mathbb{R}^{2}\right)^{2}$ and source term $m \in \mathbb{R}$.

Similarly as in Theorem 3.2, we thus obtain that system (3.69) is controllable at time $T>0$ if and only if there exists a constant $C$ such that for all $\left(\varphi_{0}, \varphi_{1}, w_{0}, w_{1}\right) \in H^{1}(\mathcal{O}) / \mathbb{R} \times L^{2}(\mathcal{O}) \times\left(\mathbb{R}^{2}\right)^{2}$ and $m \in \mathbb{R}$, the solution $(\varphi, w)$ of $(3.69)$ satisfies

$$
\left\|\left(\varphi_{0}, \varphi_{1}, w_{0}, w_{1}\right)\right\|_{H^{1}(\mathcal{O}) / \mathbb{R} \times L^{2}(\mathcal{O}) \times\left(\mathbb{R}^{2}\right)^{2}}+|m| \leq C\left\|\varphi^{\prime}\right\|_{L^{2}((0, T) \times \mathcal{C})} .
$$

Based on this equivalence, one easily checks that Theorem 3.12 with the same assumptions also holds for the controlled system (3.68) as $\varphi^{\prime}$ still solves the wave equation $\left(\varphi^{\prime}\right)^{\prime \prime}-\Delta\left(\varphi^{\prime}\right)=0$ in $\mathcal{O}$. 


\section{FurTher COMMENTS AND OPEN PROBLEMS}

1. In Section 3.2, we have shown that in the radial case, system (3.7) can be completely decoupled when decomposing it into spherical harmonics. To be more precise, the modes $k \neq 1$ simply satisfy the classical wave equation

$$
\begin{cases}\varphi^{\prime \prime}-\Delta \varphi=0, & (t, x) \in(0, T) \times \mathcal{A}\left(r_{0}, R_{0}\right), \\ \varphi(t, x)=0, & (t, x) \in(0, T) \times S\left(0, R_{0}\right), \\ \partial_{n} \varphi(t, x)=0, & (t, x) \in(0, T) \times S\left(0, r_{0}\right), \\ \left(\varphi(0, \cdot), \varphi^{\prime}(0, \cdot)\right)=\left(\varphi_{0}, \varphi_{1}\right), & \end{cases}
$$

Therefore, if for some $\theta_{0} \in(0, \pi)$, the control region is of the form

$$
\mathcal{C}=\left\{(r, \theta), \quad r \in\left(R_{1}, R_{0}\right),|\theta| \leq \theta_{0}\right\}
$$

we can construct a solution which concentrates on the ray $\theta=\pi, r \in\left(r_{0}, R\right)$ and violates the observability inequality (1.13). We refer to [17] (see also [3]) for such an explicit construction. Note that since the Hilbert Uniqueness Method proves the equivalence between the null-controllability property for (1.4) and the observability of the adjoint system (1.12), see [13], this counterexample to observability also shows that the corresponding controlled system is not null-controllable.

2. The above counterexample seems to indicate that the case of two structures in the domain $\Omega$ and of a control set on the outer boundary $\partial \Omega$ is non-controllable. Indeed, we conjecture that the required observability inequality could then be disproved by the existence of a suitable solution of the adjoint equation localized around the ray trapped between the two structures. But such construction should be done carefully due to the non-trivial boundary conditions on the fluid-solid interface.

3. Note that system (1.4) fits the abstract setting stated in [9]. Hence, similarly as for the wave equation, provided the localizing function $\chi_{\mathcal{C}}$ is smooth, the controls constructed by the Hilbert Uniqueness Method preserves the regularity of the data to be controlled, see [9] for precise statements. We expect this to be relevant in particular when trying to adapt our results to more complex settings involving, for instance, nonlinearities (see [7]) or discrete controls (see [10]).

4. Except for Section 2, our results all concern the case $\omega=B\left(0, r_{0}\right)$. Indeed, due to our strategy, our results do not apply when the structure $\omega$ is not a disk. In particular, even when $\omega$ is convex, in which case it is non-trapping, the controllability of system (1.4) through its outer boundary is completely open.

Acknowledgements. The authors are indebted to Belhassen Dehman for fruitful discussions and suggestions which significantly improve the first version of this work. The authors thank the anonymous referees for their helpful comments on the previous version of this work.

\section{REFERENCES}

[1] C. Bardos, G. Lebeau and J. Rauch, Un exemple d'utilisation des notions de propagation pour le contrôle et la stabilisation de problèmes hyperboliques. Nonlinear hyperbolic equations in applied sciences. Rend. Sem. Mat. Univ. Politec. Torino, (Special Issue) 1988 (1989) 11-31.

[2] C. Bardos, G. Lebeau and J. Rauch, Sharp sufficient conditions for the observation, control and stabilization of waves from the boundary. SIAM J. Control Optim. 30 (1992) 1024-1065.

[3] N. Burq, Mesures semi-classiques et mesures de défaut. Séminaire Bourbaki, Vol. 1996/97. Astérisque, (245): Exp. No. 826 (1997) 167-195.

[4] N. Burq and P. Gérard, Condition nécessaire et suffisante pour la contrôlabilité exacte des ondes. C. R. Acad. Sci. Paris Sér. I Math. 325 (1997) 749-752.

[5] N. Burq and M. Zworski, Geometric control in the presence of a black box. J. Amer. Math. Soc. 17 (2004) $443-471$.

[6] C. Conca, J. Planchard and M. Vanninathan, Fluids and periodic structures, vol. 38 of RAM: Res. Appl. Math. John Wiley \& Sons Ltd., Chichester (1995).

[7] B. Dehman and G. Lebeau, Analysis of the HUM control operator and exact controllability for semilinear waves in uniform time. SIAM J. Control Optim. 48 (2009) 521-550.

[8] S. Ervedoza, Spectral conditions for admissibility and observability of wave systems: applications to finite element schemes. Numer. Math. 113 (2009) 377-415. 
[9] S. Ervedoza and E. Zuazua. A systematic method for building smooth controls for smooth data. Discrete Contin. Dyn. Syst. Ser. B 14 (2010) 1375-1401.

[10] S. Ervedoza and E. Zuazua, The wave equation: Control and numerics. Control Partial Differ. Eqs. Lect. Notes Math., CIME Subseries. edited by P.M. Cannarsa and J.M. Coron. Springer Verlag (2011).

[11] P. Gérard, Microlocal defect measures. Commun. Partial Differ. Eqs. 16 (1991) 1761-1794.

[12] L. Hörmander, The analysis of linear partial differential operators. I, Distribution theory and Fourier analysis. Vol. 256 of Grundlehren der Mathematischen Wissenschaften [Fundamental Principles of Mathematical Sciences]. Springer-Verlag, Berlin, 2nd edn. (1990).

[13] J.-L. Lions, Contrôlabilité exacte, Stabilisation et Perturbations de Systèmes Distribués. Tome 1. Contrôlabilité exacte, vol. 8 RMA. Masson (1988).

[14] J.-L. Lions, Exact controllability, stabilization and perturbations for distributed systems. SIAM Review 30 (1988) 1-68.

[15] R.B. Melrose and J. Sjöstrand, Singularities of boundary value problems. II. Commun. Pure Appl. Math. 35 (1982) $129-168$.

[16] L. Miller, Controllability cost of conservative systems: resolvent condition and transmutation. J. Funct. Anal. 218 (2005) 425-444.

[17] J.V. Ralston, Solutions of the wave equation with localized energy. Commun. Pure Appl. Math. 22 (1969) 807-823.

[18] J.-P. Raymond and M. Vanninathan, Exact controllability in fluid-solid structure: the Helmholtz model. ESAIM: COCV 11 (2005) 180-203.

[19] J.-P. Raymond and M. Vanninathan, Null controllability in a fluid-solid structure model. J. Differ. Eqs. 248 (2010) $1826-1865$.

[20] M. Tucsnak and M. Vanninathan, Locally distributed control for a model of fluid-structure interaction. Systems Control Lett. 58 (2009) 547-552.

[21] M. Tucsnak and G. Weiss, Observation and Control for Operator Semigroups, vol. XI of Birkäuser Advanced Texts. Springer (2009).

[22] E. Zuazua, Exact controllability for semilinear wave equations in one space dimension. Ann. Inst. Henri Poincaré Anal. Non Linéaire 10 (1993) 109-129. 